Preprint typeset in JHEP style - HYPER VERSION

$\mathrm{TIFR} / \mathrm{TH} / 09-01$

\title{
Hydrodynamics from the D1-brane
}

\author{
Justin R. David ${ }^{* a}$, Manavendra Mahato ${ }^{b}$ and Spenta R. Wadia ${ }^{b, c}$ \\ ${ }^{a}$ Centre for High Energy Physics, Indian Institute of Science, \\ Bangalore 560012, India. \\ ${ }^{b}$ Department of Theoretical Physics, Tata Institute of Fundamental Research, \\ Homi Bhabha Road, Mumbai 40005, India. \\ ${ }^{c}$ International Centre for Theoretical Sciences, TIFR, \\ Homi Bhabha Road, Mumbai 40005, India. \\ justin@cts.iisc.ernet.in, manav@theory.tifr.res.in, \\ wadia@theory.tifr.res.in
}

Abstract: We study the hydrodynamic properties of strongly coupled $S U(N)$ Yang-Mills theory of the D1-brane at finite temperature in the framework of gauge/gravity duality. The only non-trivial viscous transport coefficient in $1+1$ dimensions is the bulk viscosity. We evaluate the bulk viscosity by isolating the quasi-normal mode corresponding to the sound channel for the gravitational background of the D1-brane. We find that the ratio of the bulk viscosity to the entropy density to be $1 / 4 \pi$. This ratio continues to be $1 / 4 \pi$ also in the regime when the D1brane Yang-Mills theory is dual to the gravitational background of the fundamental string. Our analysis shows that this ratio is equal to $1 / 4 \pi$ for a class of gravitational backgrounds dual to field theories in $1+1$ dimensions obtained by considering D1-branes at cones over Sasaki-Einstein 7-manifolds.

*On lien from Harish-Chandra Research Institute, Allahabad. 


\section{Contents}

1. Introduction 1

2. Gauge/gravity duality for the D1-brane 4

3. Hydrodynamics and the sound mode in $1+1$ dimensions 6

3.1 Lorentz structure of the correlators 6

3.2 Poles in the correlators 8

4. The sound channel in gravity 10

4.1 Linearized equations of motion for the perturbations 11

4.2 Diffeomorphism invariant sound mode 12

4.3 The dispersion relation of the sound mode 15

4.4 Holographic evaluation of the stress tensor correlators 16

5. The ratio of bulk viscosity to entropy density 19

$5.1 \xi / s$ using dispersion relation of the sound mode 19

$5.2 \xi / s$ using the Kubo's formula 21

5.3 Universality of the ratio $\xi / s \quad 22$

6. Conclusions 25

A. Consistency of the constraint equations 26

B. Identities from background field equations 29

\section{Introduction}

Recent studies of gauge theories using the framework of the AdS/CFT correspondence has revealed that gauge theories at large 't Hooft coupling and at long-distances and low-frequencies can be described by fluid mechanics 1 , 2, 3, 4, 5, 6, 7] ${ }^{1}$. A hydrodynamic description implies that the correlation functions of components of stress-energy tensor or conserved currents are fixed once a few transport coefficients are known. These transport coefficients have been evaluated for several examples of gauge theories at strong coupling using the AdS/CFT correspondence. Recently,

\footnotetext{
${ }^{1}$ For a review and a complete list of references please see [8]
} 
transport coefficients for non-linear hydrodynamics have been obtained [9, 10]. These studies indicate that for field theories which admit a gravity dual, the ratio of shear viscosity $\eta$ to the entropy density $s$ at strong t'Hooft coupling is universal [5, 11] and is given by

$$
\frac{\eta}{s}=\frac{1}{4 \pi}
$$

Most studies of this ratio have been focused on asymptotic anti-de Sitter backgrounds in various dimensions. The gauge/gravity correspondence also applies to stacks of D $p$-branes for arbitrary $p[12,13]^{2}$. It was shown in [5, 15] that the ratio of shear viscosity to entropy density for theories on Dp-branes for $p \geq 2$ also is $1 / 4 \pi^{3}$. An investigation of transport coefficients for the theory on the D1-brane is missing in the literature.

In this paper, we begin a study of the hydrodynamic behaviour of field theories in $1+1$ dimensions which admit a gravity dual. What makes one spatial dimension special is the absence of shear. In fact, for the conformal field theories in $1+1$ dimensions, there are no transport coefficients. This is because there are no non-trivial components of the symmetric traceless stress-energy tensor in these dimensions. The stress tensor is that of a perfect fluid. Thus, to study non-trivial transport properties of field theories in $1+1$ dimensions, it is necessary to study the non-conformal case. The only viscous transport coefficient for non-conformal field theories in $1+1$ dimensions is the bulk viscosity. The simplest example of such a non-conformal field theory is the theory on the D1-brane, the $1+1$ dimensional $S U(N)$ gauge theory with 16 supersymmetries. It can be obtained as a dimensional reduction of $\mathcal{N}=4$ SYM from $3+1$ dimensions. We consider this theory at finite temperature. It admits dual gravity descriptions in two regimes

$$
\begin{aligned}
& \text { (i) } \sqrt{\lambda} N^{-2 / 3}<<T<<\sqrt{\lambda}, \quad \text { and } \\
& \text { (ii) } \sqrt{\lambda} N^{-1}<<T<<\sqrt{\lambda} N^{-2 / 3} \text {. }
\end{aligned}
$$

Here, the 't Hooft coupling is denoted by $\lambda=g_{Y M}^{2} N$ and $T$ is the temperature. In regime $(i)$, the gravity dual is the background of that of the non-extremal D1-brane, while in regime $(i i)$, the dual is that of non-extremal fundamental string.

One reason why transport coefficients were not studied for backgrounds corresponding to the D1-brane is that the gauge invariant fluctuations of supergravity fields found for the case of $\mathrm{D} p$ brane with $p \geq 2$ cannot be extended to $p=1$. In this paper, we isolate the gauge invariant fluctuation of the graviton and the dilaton which corresponds to the sound channel. From its equation of motion, we find the following dispersion relation for its quasinormal mode

$$
\omega=\frac{q}{\sqrt{2}}-\frac{i}{8 \pi T} q^{2} .
$$

\footnotetext{
${ }^{2}$ For a review and recent developments on holography for the non-conformal case, please see 14 .

${ }^{3}$ Other non-conformal systems were studied in [16, 17, 18]
} 
This dispersion relation for the quasinormal mode remains the same for both the D1-brane background as well as for the F1-string background. We show that the retarded two point functions of components of stress tensor has a pole at the value of $\omega$ corresponding to the sound mode. From the universal properties of hydrodynamics in $1+1$ dimension, we find that the pole is given by

$$
\omega=v_{s} q-i \frac{\xi}{2(\epsilon+P)} q^{2} .
$$

Here, $v_{s}$ is the speed of sound in the medium, $\epsilon$ the energy density and $P$ the pressure. By comparing the dispersion relation (1.3) and the above equation, we can read out the following properties of the D1-brane gauge theory in both the regimes given in (1.2).

$$
v_{s}=\frac{1}{\sqrt{2}}, \quad \frac{\xi}{s}=\frac{1}{4 \pi},
$$

where $\xi$ is the bulk viscosity and $s$ is the entropy density. As a cross check of our calculations we use the Kubo's formula to evaluate the bluk viscosity and show that the ratio $\xi / s$ is given by $1 / 4 \pi$.

It is curious that the ratio of the bulk viscosity to entropy density has the same value as that of ratio $\eta / s$ for strongly coupled field theories which admit gravity duals in higher dimensions. Also the fact that the ratio $\xi / s$ remains $1 / 4 \pi$ for both the D1-brane and the F1-string suggests that this ratio might be universal for a class of gravitational backgrounds. We show that the this ratio continues to be $1 / 4 \pi$ for the class of $1+1$ dimensional non-conformal field theories on D1-branes at cones over Sasaki-Einstein 7-manifolds.

The organization of the paper is as follows: In the next section, we briefly review the gauge/gravity correspondence and thermodynamics for the case of the D1-brane. In section 3, we discuss the implications of hydrodynamics on the thermal Green's functions in $1+1$ dimensions. In section 4 , we discuss the details of how to isolate the gauge invariant fluctuation of the dilaton and the graviton corresponding to the sound channel. We then derive the dispersion relation of the quasi normal mode for the sound channel. We also evaluate the two point functions of the stress tensor components from gravity and show that the Lorentz structure and the structure of its pole agrees with that predicted from general considerations of hydrodynamics in $1+1$ dimensions. In section 5 , we show that the ratio of $\xi / s$ is $1 / 4 \pi$ for both the D1brane background and that of the fundamental string. This is done in two ways: we match the dissipative part of the quasi-normal mode for the sound channel in gravity to that expected from general hydrodynamic considerations and more directly by applying the Kubo formula for bulk viscosity in terms of stress tensor correlators. Both methods yield the ratio of bulk viscosity to entropy density to be $1 / 4 \pi$. We then show that this ratio continues to be $1 / 4 \pi$ for the class of field theories in $1+1$ dimensions dual to D1-branes at cones over Sasaki-Einstein manifolds. Appendix A. 
shows that the constraints we impose on the graviton and the dilaton perturbations of the D1-brane background are consistent with their equations of motion. Appendix B. contains proofs of the two identitites which are used in our derivation of the equation for the sound mode.

\section{Gauge/gravity duality for the D1-brane}

In this section, we briefly review the statement of gauge/gravity duality for the case of $N$ D1-branes. This enables us to set the notations and conventions that we will use and also to state the bounds on temperatures for which the bulk viscosity evaluated holographically from the gravity background can be trusted.

In [13], it was argued that $S U(N)$ Yang-Mills with 16 supercharges in $1+1$ dimensions at large $N$ is dual to the near horizon supergravity solution of D1-branes. The near horizon supergravity solution in the Einstein frame is given by

$$
\begin{aligned}
d s_{10}^{2} & =H^{-\frac{3}{4}}(r)\left(-d t^{2}+d x_{1}^{2}\right)+H^{\frac{1}{4}}(r)\left(d r^{2}+r^{2} d \Omega_{7}^{2}\right), \\
e^{\phi(r)} & =H(r)^{\frac{1}{2}}, \\
* F_{3}^{R R} & =6 L^{6} \omega_{S_{7}},
\end{aligned}
$$

where

$$
H(r)=\left(\frac{L}{r}\right)^{6}, \quad \text { and } L^{6}=g_{Y M}^{2} 2^{6} \pi^{3} N \alpha^{\prime 4},
$$

and $d \Omega_{7}^{2}$ refers to the metric on the unit 7 -sphere and $\omega_{S_{7}}$ its volume form. Note that, we have dualised here the Ramond-Ramond charge of the D1-brane. The gravity description is valid in the energy domain

$$
g_{Y M} N^{\frac{1}{6}}<<U<<g_{Y M} \sqrt{N}
$$

Here $U$ sets the energy scale. Note that the Yang-Mills coupling in $1+1$ dimensions has the units of energy. For completeness, we mention that the background in (2.1) is the solution of type IIB supergravity equations of motion in 10 dimensions obtained from the following action

$$
S_{I I B}=\frac{1}{16 \pi G_{10}} \int d^{10} x \sqrt{-g}\left[R(g)-\frac{1}{2} \partial_{M} \phi \partial^{M} \phi-\frac{1}{2 \cdot 3 !} e^{\phi}\left(F_{3}^{(R R)}\right)^{2}\right] .
$$

At both ends of the domain in (2.3), the curvatures of the supergravity solution in (2.1) grow and the solution breaks down. In the UV i.e. $U>>g_{Y M} \sqrt{N}$, one can trust the perturbative description of Yang-Mills theory at any value of $N$. However in the domain

$$
g_{Y M}<<U<<g_{Y M} N^{\frac{1}{6}}
$$


the dual description is given by the near horizon geometry of the fundamental string solution. This background is obtained by performing a S-duality transformation on the D-brane background in (2.1)

$$
\begin{aligned}
d s_{10}^{2} & =H^{-\frac{3}{4}}(r)\left(-d t^{2}+d x_{1}^{2}\right)+H^{\frac{1}{4}}(r)\left(d r^{2}+r^{2} d \Omega_{7}^{2}\right), \\
e^{\phi(r)} & =H(r)^{-\frac{1}{2}}, \\
* F_{3}^{N S} & =6 L^{6} \omega_{S_{7}} .
\end{aligned}
$$

Note that the only changes in the background compared to that of the D1-brane is $\phi \rightarrow-\phi$ and the Ramond-Ramond flux replaced by the Neveu-Schwarz flux on the 7 -sphere. The above background is a solution to the equations of motion from the following action

$$
S_{I I B}=\frac{1}{16 \pi G_{10}} \int d^{10} x \sqrt{-g}\left[R(g)-\frac{1}{2} \partial_{M} \phi \partial^{M} \phi-\frac{1}{2 \cdot 3 !} e^{-\phi}\left(F_{3}^{(N S)}\right)^{2}\right] .
$$

Unlike the case of the AdS/CFT duality, the supergravity solution in (2.1) and (2.6) is not asymptotically $\mathrm{AdS}_{3}$ but only conformal to $\mathrm{AdS}_{3}$ [12]. Finally, deep in the IR i.e. $U<<g_{Y M}$, the valid description is given in terms of the conformal field theory on the orbifold $\left(R^{8}\right)^{N} / S_{N}$ for any $N$. Thus both in the UV and in the IR, the $1+1$ dimensional super Yang-Mills flows to a conformal field theory. It is only in the domain $g_{Y M}<<U<<g_{Y M} \sqrt{N}$ and in the limit of large $N$, one has a dual description in terms of a supergravity solution.

To study hydrodynamics of the D1-brane theory, we need to consider the theory at finite temperature. The dual description is given in terms of the near horizon geometry of the non-extremal D1-brane solution which is given by

$$
\begin{aligned}
d s_{10}^{2} & =H^{-\frac{3}{4}}(r)\left(-f(r) d t^{2}+d x_{1}^{2}\right)+H^{\frac{1}{4}}(r)\left(\frac{d r^{2}}{f(r)}+r^{2} d \Omega_{7}^{2}\right), \\
e^{\phi(r)} & =H(r)^{\frac{1}{2}}, \\
F_{7}^{R R} & =6 L^{6} \omega_{S_{7}},
\end{aligned}
$$

where

$$
f(r)=1-\left(\frac{r_{0}}{r}\right)^{6} .
$$

The temperature and the entropy density of the D1-brane theory is related to the non-extremal parameter $r_{0}$ by

$$
T=\frac{3 r_{0}^{2}}{2 \pi L^{3}}, \quad s=\frac{1}{4 G_{3}}\left(\frac{r_{0}}{L}\right)^{4}=\frac{2 \pi^{4}}{4 ! G_{10}} r_{0}^{4} L^{3} .
$$

We can convert the domain of the validity of the D1-brane solution to ranges in temperature by identifying $U_{0}=r_{0} / \alpha^{\prime}$ with the $U$ in (2.3), using the defintion of $L$ in (2.2) and the following relations.

$$
g_{Y M}^{2}=\frac{g_{s}}{2 \pi \alpha^{\prime}}, \quad G_{10}=2^{3} \pi^{6} g_{s}^{2} \alpha^{\prime 4} .
$$


We obtain

$$
\sqrt{\lambda} N^{-\frac{2}{3}}<<T<<\sqrt{\lambda}
$$

Here, we have defined the t'Hooft coupling $\lambda=g_{Y M}^{2} N$. It is now clear that for large $N$, this is a sufficiently large domain. Now for $T<<\sqrt{\lambda} N^{-2 / 3}$, the holographic dual of the Yang-Mills theory is given by the non-extremal fundamental string solution.

$$
\begin{aligned}
d s_{10}^{2} & =H^{-\frac{3}{4}}(r)\left(-f(r) d t^{2}+d x_{1}^{2}\right)+H^{\frac{1}{4}}(r)\left(\frac{d r^{2}}{f(r)}+r^{2} d \Omega_{7}^{2}\right), \\
e^{\phi(r)} & =H(r)^{-\frac{1}{2}} \\
F_{7}^{N S} & =6 L^{6} \omega_{S_{7}} .
\end{aligned}
$$

Again writing the domain in (2.5) in terms of temperature and the 't Hooft coupling, we obtain that the above solution can be trusted in the following temperature range

$$
\sqrt{\lambda} N^{-1}<<T<<\sqrt{\lambda} N^{-\frac{2}{3}}
$$

To conclude, for very high temperatures $T>>\sqrt{\lambda}$ and for very low temperatures $T<<\sqrt{\lambda} N^{-1}$, the Yang-Mills theory flows to a free conformal field theory.

In this paper we evaluate the bulk viscosity $\xi$ using the holographic description for temperatures in the regime $\sqrt{\lambda} N^{-1}<<T<<\sqrt{\lambda}$. From the above discussion, we see that for $T>>\sqrt{\lambda}$ and $T<<\sqrt{\lambda}$, the theory flows to a conformal field theory. Therefore, we expect the bulk viscosity to vanish in these domains. However, in the regimes (2.12) and (2.14), we will see that we obtain a non-trivial value of the bulk viscosity.

\section{Hydrodynamics and the sound mode in $1+1$ dimensions}

In this section, we will discuss generalities of relativistic hydrodynamics in $1+1$ dimensions. As mentioned in the introduction, hydrodynamics in $1+1$ dimensions is special due to the absence of shear. We discuss here the constraints of conservation laws and hydrodynamics on the structure of the thermal Green's function of the stress tensor in $1+1$ dimensions. We show that this implies that the only hydrodynamic mode is longitudinal and we will determine its dispersion relation.

\subsection{Lorentz structure of the correlators}

We show using translational invariance and conservation of the stress tensor that its Green's function is entirely characterized by a single function in $1+1$ dimensions. We define the retarded Green's function of the stress energy tensor to be

$$
G_{\mu \nu, \alpha \beta}(x-y)=-i \theta\left(x^{0}-y^{0}\right)\left\langle\left[T_{\mu \nu}(x), T_{\alpha \beta}(y)\right]\right\rangle .
$$

Making use of translation invariance of the state, we can define the Fourier transform of the above correlator denoted as $G_{\mu \nu, \alpha \beta}(k)$. It is symmetric by definition in 
indices $(\mu, \nu)$ and $(\alpha, \beta)$. Further more, we have the following symmetry due to CPT invariance.

$$
G_{\mu \nu, \alpha \beta}(k)=G_{\alpha \beta, \mu \nu} .
$$

The conservation of the stress-energy tensor leads to the following Ward identity

$$
k^{\mu} G_{\mu \nu, \alpha \beta}(k)=0 .
$$

This suggests a useful tensor which forms a basis to write down the correlator is

$$
P_{\mu \nu}=\eta_{\mu \nu}-\frac{k_{\mu} k_{\nu}}{k^{2}}
$$

Note that $k^{\mu} P_{\mu \nu}=0$. If the states involved in the expectation value in (3.1) are Lorentz invariant, a convenient decomposition is to split the components into a part which contains the trace $\eta^{\mu \nu} \eta^{\alpha \beta} G_{\mu \nu, \alpha \beta}$ and the traceless. This is given by

$$
G_{\mu \nu, \alpha \beta}(k)=P_{\mu \nu} P_{\alpha \beta} G_{B}\left(k^{2}\right)+H_{\mu \nu, \alpha \beta} G_{S}\left(k^{2}\right),
$$

where

$$
H_{\mu \nu, \alpha \beta}=\frac{1}{2}\left(P_{\mu \alpha} P_{\nu \beta}+P_{\mu \beta} P_{\nu \alpha}\right)-P_{\mu \nu} P_{\alpha \beta} .
$$

Note that $\eta^{\mu \nu} H_{\mu \nu, \alpha \beta}=0$ and the two tensors in (3.5) are orthogonal; $P_{\mu \nu} P_{\alpha \beta} H_{, \alpha^{\prime} \beta^{\prime}}^{\mu \nu}=$ 0 . At this stage, it seems neccessary that one needs 2 functions to characterize the 2-point function of the stress energy tensor namely, $G_{B}$ and $G_{S}$. Now substituting explicitly the value of $k_{\mu}=(-\omega, q)$, we find that

$$
P_{t t}=\frac{q^{2}}{\omega^{2}-q^{2}}, \quad P_{t x}=\frac{\omega q}{\omega^{2}-q^{2}}, \quad P_{x x}=\frac{\omega^{2}}{\omega^{2}-q^{2}} .
$$

Using the above components of the tensor $P_{\mu \nu}$, it is easy to see that all components of $H_{\mu \nu, \alpha \beta}$ vanish. Therefore, the two point function of the stress tensor in a $1+1$ dimensional theory is entirely dependent on just one function $G_{B}\left(k^{2}\right)$. Note that due to Lorentz invariance, $G_{B}$ is a function of the Lorentz invariant quantity namely, $k^{2}$. When Lorentz invariance is broken in thermal field theory, one has rotational invariance only. For this situation, it is convenient to use the spatial projection operator $P_{\mu \nu}^{T}$ which is defined as

$$
P_{t t}^{T}=P_{t i}^{T}=P_{i t}^{T}=0, \quad P_{i j}=\delta_{i j}-\frac{k_{i} k_{j}}{\vec{k}^{2}} .
$$

But for $1+1$ dimensions, it is easy to see that this tensor vanishes identically. Therefore, $P_{\mu \nu}$ defined in (3.4) is purely longitudinal in this case. The only other projection operator in $1+1$ dimensions which is symmetric and constructed out the momenta and $\eta_{\mu \nu}$ is the

$$
\tilde{P}_{\mu \nu}=\frac{k_{\mu} k_{\nu}}{k^{2}}
$$


But tensors constructed from the above operator do not satisfy the Ward identity (3.3). Thus when Lorentz invariance is broken, one only has the following change. The arguments of the functions in $G_{B}$ and $G_{S}$ changes from the Lorentz invariant quantity $k^{2}$ to $(\omega, q)$. Thus the two point function can be written as

$$
G_{\mu \nu, \alpha \beta}(\omega, q)=P_{\mu \nu} P_{\alpha \beta} G_{B}(\omega, q)
$$

Writing it explicitly, we obtain

$$
\begin{aligned}
G_{t t, t t} & =\frac{q^{4}}{\left(\omega^{2}-q^{2}\right)^{2}} G_{B}(\omega, q), & G_{t t, t x} & =\frac{q^{3} \omega}{\left(\omega^{2}-q^{2}\right)^{2}} G_{B}(\omega, q), \\
G_{t t, x x} & =\frac{\omega^{2} q^{2}}{\left(\omega^{2}-q^{2}\right)^{2}} G_{B}(\omega, q), & G_{t x, t x} & =\frac{\omega^{2} q^{2}}{\left(\omega^{2}-q^{2}\right)^{2}} G_{B}(\omega, q), \\
G_{t x, x x} & =\frac{\omega^{3} q}{\left(\omega^{2}-q^{2}\right)^{2}} G_{B}(\omega, q), & G_{x x, x x} & =\frac{\omega^{4}}{\left(\omega^{2}-q^{2}\right)^{2}} G_{B}(\omega, q) .
\end{aligned}
$$

Thus all components of the thermal Green's function of the stress tensor are determined by a single function $G_{B}$.

\subsection{Poles in the correlators}

We show here that the function $G_{B}$, which determines the thermal Green's function must exhibit a hydrodynamic singularity due to the propagation of sound. Using Lorentz invariance, the stress tensor of a fluid in $1+1$ dimensions is given by [19]

$$
T^{\mu \nu}=(\epsilon+P) u^{\mu} u^{\nu}+P \eta^{\mu \nu}-\xi\left(u^{\mu} u^{\nu}+\eta^{\mu \nu}\right) \partial_{\lambda} u^{\lambda}
$$

where $u^{\mu}$ is the 2 -velocity with $u_{\mu} u^{\mu}=-1$ and $\xi$ is the bulk viscosity. To obtain the linearized hydrodynamic equations, consider small fluctuations from the rest frame of the fluid. We then have the following

$$
\begin{aligned}
& T^{00}=\epsilon+\delta T^{00}, \quad T^{0 x}=\delta T^{0 x}, \quad T^{x x}=P+\delta T^{x x}, \\
& u^{0}=1, \quad u^{x}=\delta u^{x} .
\end{aligned}
$$

Note that $u^{0}=1$ up to the linear order due to the constraint $u^{\mu} u_{\mu}=-1$. From the form of the stress tensor in (3.12), we can obtain the spatial variation of the velocity in terms of the stress tensor to linear order

$$
\partial_{x} \delta u^{x}=\frac{\partial_{x} \delta T^{0 x}}{\epsilon+P}
$$

Substituting this for the velocity in $T^{x x}$, we obtain to the linear order

$$
\begin{aligned}
T^{x x} & =P+\delta T^{x x}, \\
& =P-\frac{\xi}{\epsilon+P} \partial_{x} \delta T^{0 x} .
\end{aligned}
$$


The hydrodynamic equations are $\partial_{\mu} T^{\mu \nu}=0$. These reduce to

$$
\partial_{0} T^{00}+\partial_{x} T^{x 0}=0, \quad \partial_{0} T^{0 x}+\partial_{x} T^{x x}=0 .
$$

Substituting the form of the linearized form of the stress tensor given in (3.15), we obtain

$$
\begin{aligned}
\partial_{0} \delta T^{00}+\partial_{x} \delta T^{0 x} & =0, \\
\partial_{0} \delta T^{0 x}+\frac{\partial P}{\partial \epsilon} \partial_{x} \delta T^{00}-\frac{\xi}{\epsilon+P} \partial_{x}^{2} \delta T^{0 x} & =0 .
\end{aligned}
$$

Here we have used the fact that the thermodynamic variable $P$ depends only on the energy density $\epsilon$ by some equation of state. Performing the Fourier transform of the above equations in position and time, we obtain the following algebraic set of equations for $\delta T^{00}$ and $\delta T^{0 x}$

$$
\begin{aligned}
-\omega \delta T^{00}+q \delta T^{0 x} & =0, \\
-i \omega \delta T^{0 x}+i q v_{s}^{2} \delta T^{00}+q^{2} \frac{\xi}{\epsilon+P} \delta T^{0 x} & =0,
\end{aligned}
$$

where we have defined the speed of sound $v_{s}$ as

$$
v_{s}^{2}=\frac{\partial P}{\partial \epsilon} .
$$

Eliminating $\delta T^{00}$ using the first equation, we obtain the following equation for fluctuations in $\delta T^{0 x}$.

$$
\left(-i \omega^{2}+i q^{2} v_{s}^{2}+\frac{\xi}{\epsilon+P} \omega q^{2}\right) \delta T^{0 x}=0 .
$$

Therefore the fluctuation in $\delta T^{0 x}$ obey the dispersion with $\omega$ given by

$$
\omega^{2}-v_{s}^{2} q^{2}+i \frac{\xi}{\epsilon+P} \omega q^{2}=0 .
$$

Solving this to the leading order, we obtain the following dispersion relation for this longitudinal mode which we call the sound mode.

$$
\omega= \pm v_{s} q-i \frac{\xi}{2(\epsilon+P)} q^{2} .
$$

From the equations in (3.15) and (3.18), it can be seen that the remaining fluctuations also obey the same dispersion relation. This implies by the usual arguments of linear response theory [20] that the two point function of the components of the stress tensor has a pole at the above value of $\omega$. Thus we find the function $G_{B}$ in the retarded correlation functions has a pole at (3.22). 


\section{The sound channel in gravity}

As discussed in the earlier section, the D1-brane theory admits a holographic gravity dual for the ranges in temperatures given in (2.12) and (2.14). In this section, we first study the gravity solution of the D1-brane and isolate a diffeomorphism invariant perturbation which we identify as the longitudinal mode corresponding to the sound mode. From the analysis of its equation of motion and by imposing quasi-normal mode boundary conditions, we derive its dispersion relation. We then evaluate the two point function of the components of the stress tensor of the D1brane theory holographically and confirm that it has the structure predicted by the general properties of hydrodynamics in previous section.

To simplify our analysis, we first consistently truncate the 10 dimensional near horizon geometry of the D1-brane in (2.8) to 3 dimensions by dimensionally reducing on the 7 -sphere using the following ansatz.

$$
\begin{aligned}
d s_{10}^{2} & =e^{-14 B(r)} g_{\mu \nu}(x) d x^{\mu} d x^{\nu}+e^{2 B(r)} L^{2} d \Omega_{7}^{2}, \\
& =e^{-14 B(r)}\left(-c_{T}^{2}(r) d t^{2}+c_{X}^{2}(r) d z^{2}+c_{R}^{2} d r^{2}\right)+e^{2 B(r)} L^{2} d S_{X_{7}}^{2}
\end{aligned}
$$

Using this ansatz in the 10-dimensional supergravity equations of motion, one obtains a set of coupled differential equations for the fields $c_{T}(r), c_{X}(r), c_{R}(r), \phi(r)$ and $B(r)$. It can be shown that on identifying

$$
B(r)=-\frac{1}{24} \phi(r)
$$

and keeping the Ramond-Ramond flux through the 7-sphere constant, one can obtain a consistent truncation of the 10-dimensional equations to effectively 3-dimensions [15, 21]. The truncated set of equations of motion can be obtained from the following Einstein-dilaton system in 3 dimensions with action

$$
S=\frac{1}{16 \pi G_{3}} \int d^{3} x \sqrt{-g}\left[R-\frac{\beta}{2} \partial_{\mu} \phi \partial^{\mu} \phi-\mathcal{P}(\phi)\right] .
$$

Here, $\beta=\frac{16}{9}$ and $G_{3}$ is a three dimensional Newton's constant. The dilaton is denoted by $\phi$ and its potential is $\mathcal{P}=-\frac{24}{L^{2}} e^{\frac{4}{3} \phi}$. The coefficient in the dilaton potential is determined from the contributions due to the background flux through the 7 -sphere and from its curvature. The equations of motion are

$$
\begin{aligned}
R_{\mu \nu} & =\frac{\beta}{2} \partial_{\mu} \phi \partial_{\nu} \phi+\mathcal{P}(\phi), \\
\square \phi & =\frac{\mathcal{P}^{\prime}(\phi)}{\beta} .
\end{aligned}
$$

The D1-brane in 10-dimension given in (2.8) reduces to

$$
\begin{aligned}
d s^{2} & =-c_{T}(r)^{2} d t^{2}+c_{X}(r)^{2} d z^{2}+c_{R}(r)^{2} d r^{2}, \\
\phi & =-3 \log \left(\frac{r}{L}\right),
\end{aligned}
$$


with the components of the metric given by

$$
c_{T}^{2}=\left(\frac{r}{L}\right)^{8} f, \quad c_{X}^{2}=\left(\frac{r}{L}\right)^{8}, \quad c_{R}^{2}=\frac{1}{f}\left(\frac{r}{L}\right)^{2},
$$

with $f=1-\frac{r_{0}^{6}}{r^{6}}$. For future reference, we write down the the equations of motion explicitly in terms of these functions:

$$
\begin{aligned}
\frac{c_{X}^{\prime \prime}}{c_{X}}-\frac{c_{X}^{\prime}}{c_{X}} \frac{c_{R}^{\prime}}{c_{R}}+\frac{\beta}{4} \phi^{\prime 2}+\frac{c_{R}^{2} \mathcal{P}}{2} & =0, \\
\frac{c_{T}^{\prime \prime}}{c_{T}}-\frac{c_{T}^{\prime}}{c_{T}} \frac{c_{R}^{\prime}}{c_{R}}+\frac{\beta}{4} \phi^{\prime 2}+\frac{c_{R}^{2} \mathcal{P}}{2} & =0, \\
c_{R}^{2} \mathcal{P}+2 \frac{c_{T}^{\prime}}{c_{T}} \frac{c_{X}^{\prime}}{c_{X}}-\frac{\beta}{2} \phi^{\prime 2} & =0, \\
\phi^{\prime \prime}+\phi^{\prime} \ln ^{\prime}\left(\frac{c_{T} c_{X}}{c_{R}}\right) & =\frac{c_{R}^{2} \mathcal{P}^{\prime}}{\beta} .
\end{aligned}
$$

\subsection{Linearized equations of motion for the perturbations}

We consider very small wave like perturbations in the background of the above solution $g_{\mu \nu} \rightarrow g_{\mu \nu}+\delta g_{\mu \nu}$ and $\phi \rightarrow \phi+\delta \phi$. Due to translational invariance along the D1-brane directions, we can assume that all perturbations can be expanded using its Fourier mode. Focusing on one such mode, we have

$$
\delta g_{\mu \nu}(t, z, r)=e^{-i(\omega t-q z)} h_{\mu \nu}(r), \quad \delta \phi(t, z, r)=e^{-i(\omega t-q z)} \varphi(r) .
$$

We further parametrize the metric perturbations as

$$
h_{t t}=c_{T}^{2} H_{t t}, \quad h_{t z}=c_{X}^{2} H_{t z}, \quad h_{z z}=c_{X}^{2} H_{z z} .
$$

Following [2, 3, 22], we fix the gauge by choosing $\delta g_{r \mu}=0$. The equations of motion up to linear order in perturbations are

$$
\begin{array}{r}
H_{t t}^{\prime \prime}+\ln ^{\prime}\left(\frac{c_{T}^{2} c_{X}}{c_{R}}\right) H_{t t}^{\prime}-\ln ^{\prime}\left(c_{T}\right) H_{z z}^{\prime}-\frac{c_{R}^{2}}{c_{T}^{2}} Z_{0}-2 c_{R}^{2} \frac{\partial \mathcal{P}}{\partial \phi} \varphi=0 \\
H_{t z}^{\prime \prime}+\ln ^{\prime}\left(\frac{c_{X}^{3}}{c_{T} c_{R}}\right) H_{t z}^{\prime}=0 \\
H_{z z}^{\prime \prime}+\ln ^{\prime}\left(\frac{c_{T} c_{X}^{2}}{c_{R}}\right) H_{z z}^{\prime}-H_{t t}^{\prime} \ln ^{\prime}\left(c_{x}\right)+\frac{c_{R}^{2}}{c_{T}^{2}} Z_{0}+2 c_{R}^{2} \frac{\partial \mathcal{P}}{\partial \phi} \varphi=0 \\
\varphi^{\prime \prime}+\ln ^{\prime} \frac{c_{T} c_{X}}{c_{R}} \varphi^{\prime}+c_{R}^{2}\left(\frac{\omega^{2}}{c_{T}^{2}}-\frac{q^{2}}{c_{X}^{2}}\right) \varphi+\frac{1}{2} \phi^{\prime}\left(H_{z z}^{\prime}-H_{t t}^{\prime}\right)-\frac{c_{R}^{2}}{\beta} \frac{\partial^{2} \mathcal{P}}{\partial \phi^{2}} \varphi=0
\end{array}
$$

where $A_{t}=q^{2} \frac{c_{T}^{2}}{c_{X}^{2}}$ and $Z_{0}=A_{t} H_{t t}+2 q \omega H_{t z}+\omega^{2} H_{z z}$. We also obtain following first order constraints from Einstein equations for $\delta R_{r \mu}$.

$$
H_{z z}^{\prime}+\frac{q}{\omega} H_{t z}^{\prime}-\frac{1}{\omega^{2}} A_{t} \ln ^{\prime} \frac{c_{X}}{c_{T}} H_{t t}+\frac{1}{\omega^{2}} \ln ^{\prime} \frac{c_{X}}{c_{T}} Z_{0}+\beta \phi^{\prime} \varphi=0,
$$




$$
\begin{aligned}
H_{t t}^{\prime}-\ln ^{\prime}\left(\frac{c_{X}}{c_{T}}\right) H_{t t}+\frac{\omega c_{X}^{2}}{q c_{T}^{2}} H_{t z}^{\prime}-\beta \phi^{\prime} \varphi & =0, \\
\ln ^{\prime}\left(c_{T}\right) H_{z z}^{\prime}-\ln ^{\prime}\left(c_{X}\right) H_{t t}^{\prime}-\beta \phi^{\prime} \varphi^{\prime}+\frac{c_{R}^{2}}{c_{T}^{2}} Z_{0}+c_{R}^{2} \frac{\partial \mathcal{P}}{\partial \phi} \varphi & =0 .
\end{aligned}
$$

In the appendix $\mathrm{A}$, we show that the above 3 constaints can be consistently imposed on the 4 dynamical equations of motion.

\subsection{Diffeomorphism invariant sound mode}

Fixing the gauge $\delta g_{\mu r}=0$ does not exhaust all gauge degrees of freedom. One is left with the residual gauge freedom under the infinitesimal diffeomorphisms $x^{\mu} \rightarrow x^{\mu}+\xi^{\mu}$ with $\mu \in\{t, z, r\}$. Following the approach of [22], we will construct a quantity using the above perturbations which will be invariant under the diffeomorphism of the metric i.e. $\delta g_{\mu \nu} \rightarrow \delta g_{\mu \nu}-\nabla_{\mu} \xi_{\nu}-\nabla_{\nu} \xi_{\mu}$. The perturbations change under diffeomorphism as

$$
\begin{aligned}
\delta g_{\mu \nu} & \rightarrow \delta g_{\mu \nu}-\nabla_{\mu} \xi_{\nu}-\nabla_{\nu} \xi_{\mu}, \\
\delta g_{t t} & \rightarrow \delta g_{t t}-2 \nabla_{t} \xi_{t} \rightarrow \delta g_{t t}+2 i \omega \xi_{t}+\frac{\left(c_{T}^{2}\right)^{\prime}}{c_{R}^{2}} \xi_{r}, \\
\delta g_{t z} & \rightarrow \delta g_{t z}+i \omega \xi_{z}+i q \xi_{t} \\
\delta g_{z z} & \rightarrow \delta g_{z z}-2 i q \xi_{z}-\frac{\left(c_{X}^{2}\right)^{\prime}}{c_{R}^{2}} \xi_{r} .
\end{aligned}
$$

Then, the combination $Z_{0}=A_{t} H_{t t}+2 q \omega H_{t z}+\omega^{2} H_{z z}$ changes as

$$
\begin{aligned}
Z_{0} & \rightarrow Z_{0}+\frac{q^{2}}{c_{X}^{2}}(2 i \omega) \xi_{t}+\frac{q^{2}}{c_{X}^{2}} \frac{\left(c_{T}^{2}\right)^{\prime}}{c_{R}^{2}} \xi_{r}+\frac{2 q \omega}{c_{X}^{2}}\left(i \omega \xi_{z}-i q \xi_{t}\right)-\frac{\omega^{2}}{c_{X}^{2}}\left(2 i q \xi_{z}+\frac{\left(c_{X}^{2}\right)^{\prime}}{c_{R}^{2}} \xi_{r}\right), \\
\delta Z_{0} & =\frac{2 \xi_{r} A_{H} \ln ^{\prime}\left(c_{X}\right)}{c_{R}^{2}}
\end{aligned}
$$

where

$$
A_{H}=A_{t} \frac{\ln ^{\prime} c_{T}}{\ln ^{\prime} c_{X}}-\omega^{2}
$$

The dilaton also changes under diffeomorphism as

$$
\varphi \rightarrow \varphi-\partial^{\mu} \phi \xi_{\mu} \rightarrow \varphi-\frac{\phi^{\prime}}{c_{R}^{2}} \xi_{r}
$$

We find the following combination gauge invariant.

$$
Z_{P}=Z_{0}+A_{\varphi} \varphi \quad \text { where } \quad A_{\varphi}=\frac{2 A_{H} \ln ^{\prime}\left(c_{X}\right)}{\phi^{\prime}} .
$$

Note that unlike the case of higher dimensional Dp-branes with $p \geq 2$, studied by [15], there is only a single gauge invariant mode for $p=1$. Note that the $Z_{0}$ constructed 
in 15 for $p \geq 2$ cannot be trivially extended for this case as $H$ as defined in 15 does not exists for $p=1$. Here, we note that the role of $H$ for $p=1$ is played by the dilaton fluctuation. We will call the diffeomorphism invariant fluctuation $Z_{p}$ as the sound mode as it constitutes fluctuations longitudinal to the wave directions.

Next we outline the steps involved in obtaining the second order equation satisfied by this mode. From the definition, we have

$$
\begin{aligned}
Z_{P}^{\prime \prime}= & A_{t}^{\prime \prime} H_{t t}+2 A_{t}^{\prime} H_{t t}^{\prime}+A_{\varphi}^{\prime \prime} \varphi+2 A_{\varphi}^{\prime} \varphi^{\prime} \\
& +A_{t} H_{t t}^{\prime \prime}+2 q \omega H_{t z}^{\prime \prime}+\omega^{2} H_{z z}^{\prime \prime}+A_{\varphi} \varphi^{\prime \prime}
\end{aligned}
$$

where

$$
A_{t}=q^{2} \frac{c_{T}^{2}}{c_{X}^{2}} .
$$

Using equations (4.14)-(4.20), we simplify above to

$$
\begin{aligned}
Z_{P}^{\prime \prime}+ & \ln ^{\prime}\left(\frac{c_{T} c_{X}}{c_{R}}\right) Z_{P}^{\prime}-\frac{c_{R}^{2}}{c_{T}^{2}}\left(A_{t}-\omega^{2}\right) Z_{P} \\
= & H_{t t}\left[-4 A_{t}\left[\ln ^{\prime}\left(\frac{c_{T}}{c_{X}}\right)\right]^{2}+A_{t}^{\prime \prime}+A_{t}^{\prime} \ln ^{\prime}\left(\frac{c_{T} c_{X}}{c_{R}}\right)\right] \\
& +\varphi\left[4 A_{t} \ln ^{\prime}\left(\frac{c_{T}}{c_{X}}\right) \beta \phi^{\prime}+\ln ^{\prime}\left(\frac{c_{T} c_{X}}{c_{R}}\right) A_{\varphi}^{\prime}+A_{\varphi}^{\prime \prime}+2 c_{R}^{2} \mathcal{P}^{\prime}\left(A_{t}-\omega^{2}\right)+\frac{c_{R}^{2}}{\beta} \mathcal{P}^{\prime \prime} A_{\varphi}\right] \\
& +2 A_{\varphi}^{\prime} \varphi^{\prime} .
\end{aligned}
$$

Since $A_{t}^{\prime}=2 A_{t} \ln ^{\prime}\left(\frac{c_{T}}{c_{X}}\right)$,

$$
A_{t}^{\prime \prime}=4 A_{t}\left[\ln ^{\prime}\left(\frac{c_{T}}{c_{X}}\right)\right]^{2}+2 A_{t} \ln ^{\prime \prime}\left(\frac{c_{T}}{c_{X}}\right) .
$$

Evaluating the difference of the Einstein equations (4.8)-(4.9), we obtain

$$
\ln ^{\prime}\left(\frac{c_{T}}{c_{X}}\right) \ln ^{\prime}\left(\frac{c_{T} c_{X}}{c_{R}}\right)+\ln ^{\prime \prime}\left(\frac{c_{T}}{c_{X}}\right)=0 .
$$

Using equation (4.29) and (4.30), we obtain

$$
\begin{aligned}
Z_{P}^{\prime \prime}+ & \ln ^{\prime}\left(\frac{c_{T} c_{X}}{c_{R}}\right) Z_{P}^{\prime}-\frac{c_{R}^{2}}{c_{T}^{2}}\left(A_{t}-\omega^{2}\right) Z_{P} \\
= & \varphi\left[4 A_{t} \ln ^{\prime}\left(\frac{c_{T}}{c_{X}}\right) \beta \phi^{\prime}+\ln ^{\prime}\left(\frac{c_{T} c_{X}}{c_{R}}\right) A_{\varphi}^{\prime}+A_{\varphi}^{\prime \prime}+2 c_{R}^{2} \mathcal{P}^{\prime}\left(A_{t}-\omega^{2}\right)+\frac{c_{R}^{2}}{\beta} \mathcal{P}^{\prime \prime} A_{\varphi}\right] \\
& +2 A_{\varphi}^{\prime} \varphi^{\prime} .
\end{aligned}
$$

Using Einstein equations and the dilaton equation of motion (4.8)-(4.11), one can proove the following identities (see Appendix. B) .

$$
\beta \phi^{\prime}\left(A_{t}-\omega^{2}\right)+A_{\varphi}^{\prime}=-\left[\frac{\phi^{\prime \prime}}{\phi^{\prime}}+\ln ^{\prime}\left(\frac{c_{X}}{c_{T} c_{R}}\right)\right] A_{\varphi},
$$




$$
\begin{aligned}
4 A_{t} \ln ^{\prime}\left(\frac{c_{T}}{c_{X}}\right) \beta \phi^{\prime}+\ln ^{\prime}\left(\frac{c_{T} c_{X}}{c_{R}}\right) A_{\varphi}^{\prime}+A_{\varphi}^{\prime \prime} & +2 c_{R}^{2} \mathcal{P}^{\prime}\left(A_{t}-\omega^{2}\right)+\frac{c_{R}^{2}}{\beta} \mathcal{P}^{\prime \prime} A_{\varphi} \\
& =2 A_{\varphi}^{\prime}\left[\frac{c_{R}^{\prime}}{c_{R}}-\frac{\phi^{\prime \prime}}{\phi^{\prime}}\right] .
\end{aligned}
$$

Using relation (4.32), one can write the relation (A.4) as

$$
A_{\varphi}\left[\left(\frac{c_{R}^{\prime}}{c_{R}}-\frac{\phi^{\prime \prime}}{\phi^{\prime}}\right) \varphi+\varphi^{\prime}\right]=Z_{P}^{\prime}+\ln ^{\prime}\left(\frac{c_{X}}{c_{T}}\right) Z_{P}
$$

Finally using relation (4.33), the equation (4.31) can be written as

$$
Z_{P}^{\prime \prime}+\ln ^{\prime}\left(\frac{c_{T} c_{X}}{c_{R}}\right) Z_{P}^{\prime}-\frac{c_{R}^{2}}{c_{T}^{2}}\left(A_{t}-\omega^{2}\right) Z_{P}=2 A_{\varphi}^{\prime}\left[\varphi^{\prime}+\left(\frac{c_{R}^{\prime}}{c_{R}}-\frac{\phi^{\prime \prime}}{\phi^{\prime}}\right) \varphi\right] .
$$

Using the relation (4.34), we obtain

$$
Z_{P}^{\prime \prime}+\left[\ln ^{\prime}\left(\frac{c_{T} c_{X}}{c_{R}}\right)-2 \frac{A_{\varphi}^{\prime}}{A_{\varphi}}\right] Z_{P}^{\prime}-\left[\frac{c_{R}^{2}}{c_{T}^{2}}\left(A_{t}-\omega^{2}\right)+2 \frac{A_{\varphi}^{\prime}}{A_{\varphi}} \ln ^{\prime}\left(\frac{c_{X}}{c_{T}}\right)\right] Z_{P}=0
$$

This is the equation for $Z_{P}$ from which we will obtain the dispersion relation for its quasi-normal mode. Here we make the following observation regarding the equation of $Z_{P}$. Consider a minimally coupled scalar $\Psi$ in the background (4.5), its equation of motion is given by

$$
\begin{aligned}
\frac{1}{\sqrt{-g}} \partial_{\mu}\left(\sqrt{-g} g^{\mu \nu} \partial_{\nu} \Psi\right) & =0, \\
\psi^{\prime \prime}+\ln ^{\prime}\left(\frac{c_{T} c_{X}}{c_{R}}\right) \psi^{\prime}-\frac{c_{R}^{2}}{c_{T}^{2}}\left(A_{t}-\omega^{2}\right) \psi & =0,
\end{aligned}
$$

where $g_{\mu \nu}$ is the background metric in (4.5). The second line is obtained from the first by focusing on the Fourier component $\Psi(t, z, r)=e^{-i(\omega t+q z)}$. Now comparing the above equation with the equation for $Z_{P}$ we see that the the equation for $Z_{P}$ (4.36) is almost a equation for a minimally coupled scalar except for the terms that are proportional to $A_{\varphi}^{\prime} / A_{\varphi}$. Note that the dilaton dependence of the equation for $Z_{P}$ is entirely contained in these terms.

Finally we emphasize that the analysis to obtain the equation for $Z_{P}$ in (4.36) depended only on the fact that the background is of the form (4.5) with a radially dependent dilaton profile (4.6) that satisfy the equations of motion (4.8), (4.9), (4.10) and (4.11) obtained from the Lagrangian in (4.3). At each step we have not assumed any form for the functions $c_{T}, c_{X}, c_{R}, \phi$, the dilaton potential $\mathcal{P}$ and any specific value for the constant $\beta$. Therefore for any radially symmetric solution of the form (4.5) and a dilaton profile which solves the dilaton equation of motion, the equation for $Z_{P}$ is given by (4.36). 


\subsection{The dispersion relation of the sound mode}

For further analysis, we will use the explicit expressions of metric coefficients and

dilaton. We first change the variable to $Y=\frac{Z_{P}}{A_{\varphi}}$ and the independent variable to $u=\frac{r_{0}^{2}}{r^{2}}$, then the equation becomes

$$
\partial_{u}^{2} Y-\frac{\left(2+u^{3}\right)}{u\left(1-u^{3}\right)} \partial_{u} Y-\left[\frac{q^{2} L^{6}}{4 r_{0}^{4}} \frac{\left(1-\lambda-u^{3}\right)}{\left(1-u^{3}\right)^{2}}+\frac{18 u^{4}(4 \lambda-3)}{\left(1-u^{3}\right)\left(4-4 \lambda-u^{3}\right)^{2}}\right] Y=0,
$$

where $\lambda=\frac{\omega^{2}}{q^{2}}$. First we look for its solution near the horizon. For $1-u \ll 1$, the equation becomes

$$
\partial_{u}^{2} Y-\frac{1}{1-u} \partial_{u} Y+\left[\frac{\alpha^{2} \omega^{2}}{9(1-u)^{2}}+\frac{6}{(3-4 \lambda)(1-u)}\right] Y=0,
$$

where $\alpha=\frac{L^{3}}{2 r_{0}^{2}}$. To pick up the ingoing solution at the horizon, let us define $x=$ $\ln (1-u)$. Then in terms of $x$ and for $1-u<<1$, the above equation reduces to the oscillator equation

$$
\left(\partial_{x}^{2}+\frac{\alpha^{2} \omega^{2}}{9}\right) Y=0
$$

Therefore, the ingoing solution for $1-u<<1$ behaves as $e^{-\frac{i}{3} \alpha \omega x}=(1-u)^{-\frac{i}{3} \alpha \omega}$. We next consider a solution of the type

$$
Y=\left(1-u^{3}\right)^{-\frac{i}{3} \alpha \omega} Z(u) .
$$

Then the equation for $Z(u)$ is given by

$$
\begin{aligned}
\partial_{u}^{2} Z- & \frac{\partial_{u} Z}{u\left(1-u^{3}\right)}\left[2+u^{3}-2 i \alpha \omega u^{3}\right] \\
& +Z\left[\alpha^{2} \omega^{2} \frac{\left(1-u^{4}\right)}{\left(1-u^{3}\right)^{2}}-\frac{\alpha^{2} q^{2}}{\left(1-u^{3}\right)}-\frac{18 u^{4}(4 \lambda-3)}{\left(1-u^{3}\right)\left(4-4 \lambda-u^{3}\right)^{2}}\right]=0 .
\end{aligned}
$$

In this equation, all terms are dimensionless. From the definition of temperature in (2.10), we see that $\alpha \sim 1 / T$, therefore in the hydrodynamic limit

$$
\omega<<T \text { and } q<<T,
$$

we ignore terms of order $q^{2} / T^{2}, \omega^{2} / T^{2}, \omega q / T^{2}$ and higher, but keep terms of order $\omega / T, q / T$. Performing this limit in the equation for $Z$, we obtain

$$
\partial_{u}^{2} Z-\frac{\left\{2+(1-2 i \alpha \omega) u^{3}\right\}}{u\left(1-u^{3}\right)} \partial_{u} Z-\frac{18 u^{4}(4 \lambda-3)}{\left(1-u^{3}\right)\left(4-4 \lambda-u^{3}\right)^{2}} Z=0 .
$$

The well behaved solution of the above equation at $u=1$, the horizon, is given by

$$
Z=\frac{6 \lambda-2(1-\lambda)(3-4 i \alpha \omega)-u^{3}(3+2 i \alpha \omega)}{12(3-2 i \alpha \omega)\left(4-4 \lambda+u^{3}\right)} .
$$


To obtain the quasi-normal modes of this solution, we need to impose the Dirichlet condition $Z=0$ at the boundary at $u \rightarrow \infty$. This leads to the following cubic equation for $\omega$.

$$
-4 i \alpha \omega^{3}+6 \omega^{2}+4 i \alpha \omega q^{2}-3 q^{2}=0 .
$$

Solving this equation perturbatively by assuming $\omega \sim q$, we obtain the following dispersion relation for the sound mode.

$$
\omega= \pm \frac{1}{\sqrt{2}} q-i \frac{\alpha}{6} q^{2}+\ldots
$$

with

$$
\alpha=\frac{L^{3}}{2 r_{0}^{2}}=\frac{3}{4 \pi T} .
$$

\subsection{Holographic evaluation of the stress tensor correlators}

In this section, we use the standard prescription of the gauge/gravity correspondence to evaluate the stress tensor correlations. For this, we first need to expand the action in (4.3) along with the Gibbons-Hawking boundary term to second order in the fluctuation $H_{\mu \nu}$. The bulk action and the boundary term is given by

$$
\begin{aligned}
& S=S_{\text {bulk }}+S_{G H}, \\
& S=\frac{1}{16 \pi G_{3}} \int d^{3} x \sqrt{-g}\left[R-\frac{\beta}{2} \partial_{\mu} \phi \partial^{\mu} \phi-\mathcal{P}(\phi)\right]+\left.\frac{1}{8 \pi G_{3}} \int d^{2} x \sqrt{-h} K\right|_{r \rightarrow \infty} .
\end{aligned}
$$

where

$$
\beta=\frac{16}{9} \quad \mathcal{P}=-\frac{24}{L^{2}} e^{4 \phi / 3}
$$

and $h$ is the boundary metric at a large but fixed value of $r$ and $K$ is the extrinsic curvature of the boundary ${ }^{4}$. Using the equations of motion (4.14)-(4.17) and the constraints (4.18)-(4.19) we can rewrite the expansion of the bulk action to second order in the fluctuations as a total derivative in the radial coordinate. This is given by

$$
\begin{aligned}
S_{b u l k}^{(2)}= & \int d^{3} x\left[-\frac{3 c_{X}^{3}}{2 c_{T} c_{R}} H_{t z} H_{t z}^{\prime}+\frac{c_{X}^{3}}{c_{T} c_{R}}\left(\frac{c_{T}^{\prime}}{c_{T}}-2 \frac{c_{X}^{\prime}}{c_{X}}\right) H_{t z}^{2}-\frac{\beta}{2} \frac{c_{T} c_{X}}{c_{R}} \varphi \varphi^{\prime}\right. \\
& +\frac{c_{T} c_{X}}{4 c_{R}}\left(H_{t t}+H_{z z}\right)\left(H_{t t}+H_{z z}\right)^{\prime}+\frac{c_{T} c_{X}}{2 c_{R}} \ln ^{\prime}\left(c_{T} c_{X}\right) H_{t t} H_{z z} \\
& +\frac{c_{T} c_{X}}{4 c_{R}}\left\{H_{t t} H_{t t}^{\prime}+H_{z z} H_{z z}^{\prime}+\left(H_{t t}-H_{z z}\right)\left(\frac{c_{T}^{\prime}}{c_{T}} H_{t t}-\frac{c_{X}^{\prime}}{c_{X}} H_{z z}\right)\right\} \\
& \left.+\frac{\beta}{4} \frac{c_{T} c_{X}}{c_{R}}\left(H_{t t}-H_{z z}\right) \phi^{\prime} \varphi\right]^{\prime} .
\end{aligned}
$$

\footnotetext{
${ }^{4}$ In general there are counter terms one has to add to regulate the action [23, 24, 25, 14, these counter terms are crucial to regulate the one point function of the stress tensor. However for the two point functions which we will be interested in, they are not relevant.
} 
The second order perturbation in extrinsic curvature term is given by

$$
\begin{aligned}
\sqrt{-h} K= & -\frac{c_{X}^{3}}{8 c_{T} c_{R}}\left[4\left(\frac{c_{T}^{\prime}}{c_{T}} H_{t z}^{2}-3 \frac{c_{X}^{\prime}}{c_{X}} H_{t z}^{2}-2 H_{t z}^{\prime} H_{t z}\right)\right. \\
& \left.+\frac{c_{T}^{2}}{c_{X}^{2}}\left(H_{t t}+H_{z z}\right)\left(2\left(H_{t t}+H_{z z}\right)^{\prime}+\ln ^{\prime}\left(c_{T} c_{X}\right)\left(H_{t t}+H_{z z}\right)\right)\right] .
\end{aligned}
$$

Since the bulk action at second order in perturbation is just a total derivative we just have to evaluate its contribution at the boundary. Then the boundary action at second order in fluctuations including the Gibbons Hawking term then reduces to

$$
S^{(2)}=\frac{1}{16 \pi G_{3}} \int \frac{d \omega d q}{(2 \pi)^{2}} \mathcal{L}
$$

where

$$
\mathcal{L}=\frac{c_{X}^{3}}{4 \omega q c_{T} c_{R}} Z_{P}(\vec{k}) H_{t z}^{\prime}+\mathcal{L}_{\text {contact }},
$$

and $\mathcal{L}_{\text {contact }}$ represents the part of the Lagrangian without any derivatives.

$$
\begin{aligned}
\mathcal{L}_{\text {contact }}= & -\frac{c_{T} c_{X}}{4 c_{R}}\left[\ln ^{\prime}\left(c_{T} c_{X}\right) H_{t t} H_{z z}+\frac{c_{X}^{\prime}}{c_{X}} H_{t t}^{2}+\frac{c_{T}^{\prime}}{c_{T}} H_{z z}^{2}-2 \frac{q}{\omega} \ln ^{\prime}\left(\frac{c_{X}}{c_{T}}\right) H_{t z} H_{t t}\right] \\
& +\frac{c_{X}^{3}}{c_{T} c_{R}} \frac{c_{X}^{\prime}}{c_{X}} H_{t z}^{2}-\frac{c_{T} c_{X}}{4 c_{R}} \varphi\left[2 \varphi\left\{\frac{c_{R}^{2} \mathcal{P}^{\prime}}{\phi^{\prime}}-\beta \ln ^{\prime}\left(c_{T} c_{X}\right)\right\}-2 \beta \phi^{\prime}\left(H_{t t}-H_{z z}\right)\right. \\
& \left.+\frac{A_{\varphi}}{\omega^{2}} \ln ^{\prime}\left(\frac{c_{X}}{c_{T}}\right) H_{t t}+\frac{2 Z_{0}}{\phi^{\prime}}\left\{\frac{c_{R}^{2}}{c_{T}^{2}}-\frac{\ln ^{\prime} c_{T}}{\omega^{2}} \ln ^{\prime}\left(\frac{c_{X}}{c_{T}}\right)\right\}\right] .
\end{aligned}
$$

To obtain the above form of the action we have used the constraints (4.18)-(4.20) to rewrite all derivative terms in $\mathcal{L}$ in terms of the derivative $H_{t z}^{\prime}$. We can further manipulate the boundary action and reduce it to the form

$$
S^{(2)}=\frac{1}{16 \pi G_{3}} \int \frac{d \omega d q}{(2 \pi)^{2}} \mathcal{A}(\omega, q, r) Z_{P}^{\prime}(r, \vec{k}) Z_{P}(r,-\vec{k})+S_{C T}^{(2)},
$$

where

$$
\mathcal{A}(\omega, q, r)=-\frac{\beta}{2 A_{\varphi}^{2}} \frac{c_{T} c_{X}}{c_{R}} .
$$

The contact term in equation (4.53) is

$$
\begin{aligned}
S_{C T}^{(2)}= & \frac{1}{16 \pi G_{3}} \int \frac{d \omega d q}{(2 \pi)^{2}}\left[\mathcal{L}_{\text {contact }}\right]-\mathcal{A}\left[A_{t}^{\prime} H_{t t}+\left(2 A_{t}+\frac{A_{\varphi}}{2 \beta \omega^{2}}\right) \ln ^{\prime}\left(\frac{c_{X}}{c_{T}}\right) H_{t t}\right. \\
& +\varphi\left\{A_{\varphi}^{\prime}+\beta \phi^{\prime}\left(A_{t}-\omega^{2}\right)-A_{\varphi}\left(\ln ^{\prime}\left(c_{R}\right)-\frac{\phi^{\prime \prime}}{\phi^{\prime}}\right)\right\} \\
& \left.+Z_{0}\left\{-\ln ^{\prime}\left(\frac{c_{X}}{c_{T}}\right)+\frac{A_{\varphi}}{\beta \phi^{\prime}} \frac{c_{R}^{2}}{c_{T}^{2}}-\frac{A_{\varphi}}{\beta \phi^{\prime} \omega^{2}} \ln ^{\prime}\left(c_{T}\right) \ln ^{\prime}\left(\frac{c_{X}}{c_{T}}\right)\right\}\right] Z_{P}(r,-\vec{k}) .
\end{aligned}
$$

Now that we have the boundary action, we can evaluate the stress-tensor correlators by using the standard rules of the AdS/CFT correspondence. Note that in 
this case the geometry is not asymptotically AdS. However we expect the rules of the AdS/CFT correspondence to still be valid for this case since it can be related to the anti-de Sitter geometry up to a conformal factor [12, 14]. The boundary values of the fluctuation $H_{\mu \nu}$ couple to the stress tensor of the boundary theory as in [3]

$$
S_{\text {coupling }}=\frac{1}{2} \int d^{4} x\left[H_{t t}^{0} T^{t t}+H_{z z}^{0} T^{z z}+2 H_{t z}^{0} T^{t z}\right] .
$$

Here, the indices are raised, lowered and contracted using the flat metric $d s^{2}=$ $-d t^{2}+d z^{2}$. The superscript 0 indicates the fact that we are looking at the $r \rightarrow \infty$ or the $u=\frac{r_{0}^{2}}{r^{2}} \rightarrow 0$ limit of the corresponding bulk fields. Thus these correspond to the boundary values of the fluctuations $H_{\mu \nu}$. Using the above coupling and the rules of the AdS/CFT correspondence summarized in the equation

$$
\left\langle\exp \left(i S_{\text {coupling }}\right)\right\rangle=\exp \left[i S^{(2)}\left(H_{\mu \nu}^{0}\right)\right]
$$

we can evaluate the various two point functions of the components of the stress tensor. Consider the retarded two point function $G_{t t, t t}=-i\left\langle\left[T_{t t}, T_{t t}\right]\right\rangle$, using the AdS/CFT prescription we obtain

$$
G_{t t, t t}=-4 \frac{\delta S^{(2)}}{\delta H_{t t}^{0}(\vec{k}) \delta H_{t t}^{0}(-\vec{k})} .
$$

From the definition of $Z_{P}$, we note that its boundary value is related to the boundary values of the fluctuations by

$$
Z_{P}^{0}=q^{2} H_{t t}^{0}+2 \omega q H_{t z}^{0}+\omega^{2} H_{z z}^{0}+A_{\varphi}^{0} \varphi^{0} .
$$

From (1.44), we see that the expansion of $Z_{P}$ near the boundary is given by

$$
\begin{aligned}
Z_{P} & =C(1+\ldots)+D u^{3}(1+\ldots), \\
& =Z_{P}^{0}\left[1+\ldots+\frac{D}{C} \frac{r_{0}^{6}}{r^{6}}(1+\ldots)\right] .
\end{aligned}
$$

Here $C(\omega, q)$ and $D(\omega, q)$ are independent of $u$. The ellipses denote higher powers in $u=\frac{r_{0}^{2}}{r^{2}}$. Now substituting the above expansion for $Z_{P}$, in $S^{(2)}$ it is easy to see that relevant term in the boundary action is the first term in (4.53) which involves the derivative of $Z_{P}$. This results in the following expression for the two point function of the stress tensor.

$$
G_{t t, t t}=-4 \frac{\delta S^{(2)}}{\delta H_{t t}^{0}(\vec{k}) \delta H_{t t}^{0}(-\vec{k})}=-\frac{1}{16 \pi G_{3}} \frac{q^{4}}{\left(\omega^{2}-q^{2}\right)^{2}} \frac{6 r_{0}^{6}}{L^{7}} \frac{D}{C} .
$$

Comparing the above holographic result with (3.11) we see that the expected Lorentz factor for this correlator is reproduced. Furthermore we can read out the holographic value of $G_{B}$ as

$$
G_{B}(\omega, q)=-\frac{1}{16 \pi G_{3}} \frac{6 r_{0}^{6}}{L^{7}} \frac{D}{C} .
$$


The poles in the Green's function are therefore same as the zeros of the factor $C$. The Dirichlet boundary condition for the mode $Z_{P}=0$ at the horizon is equivalent to setting $C=0$ as noted for the case of backgrounds asymptotic to AdS which were studied in [22]. Thus the poles in the two point function of the stress tensor which leads to the dispersion relation of the sound mode is as given in equation (4.46). As a consistency check of our calculations, we have evaluated all the remaining two point functions of the components of the stress tensor. In each case the expected Lorentz factor given in (3.11) is reproduced with the same expression for $G_{B}(\omega, q)$.

\section{The ratio of bulk viscosity to entropy density}

In this section, we use two methods to evaluate the ratio of bulk viscosity to entropy density $\xi / s$ of the D1-brane theory in the gravity regime which is valid for temperatures in the range $\sqrt{\lambda} N^{-\frac{2}{3}}<<T<<\sqrt{\lambda}$. We use the dispersion relation of the sound mode to read out this ratio, then as a cross check we determine this ratio by evaluating the bulk viscosity directly using the Kubo's formula for the bulk viscosity in one spatial dimensions. We then show that the ratio $\xi / s$ continues to be the same for the temperatures in the range $\sqrt{\lambda} N^{-1}<<T<<\sqrt{\lambda} N^{-\frac{2}{3}}$ when the D1-brane background is replaced by the F1-string solution. In both these regimes, the ratio

$$
\frac{\xi}{s}=\frac{1}{4 \pi} .
$$

We also show that for gauge theories corresponding to D1-branes at cones over SasakiEinstein 7 -manifolds the ratio continues to be $1 / 4 \pi$.

\section{$5.1 \xi / s$ using dispersion relation of the sound mode}

Using the dispersion relation of the sound mode in gravity (4.46) and comparing it with the dispersion relation of the sound mode using general hydrodynamic consideration (3.21), we deduce the transport properties of the D1-brane matter at temperature $T$. As we have seen in section 3 , general hydrodynamics considerations give the following dispersion relation for the sound mode

$$
\omega=v_{s}-\frac{i}{2} \frac{1}{\epsilon+P} \xi q^{2}, \quad v_{s}^{2}=\frac{\partial P}{\partial \epsilon} .
$$

Here, $v_{s}$ is the speed of sound in the medium $P$, the pressure and $\epsilon$ the energy density. Now comparing this with the dispersion relation obtained from gravity in (4.46), we can read out the speed of sound in the D1-brane matter as

$$
v_{s}^{2}=\frac{1}{2} \text {. }
$$

It is now easy to see that from the definition of the speed of sound $v_{s}=\frac{\partial P}{\partial \epsilon}$, we obtain the equation of state

$$
\epsilon=2 P
$$


for the D1-brane matter. Thus the mediums seem to behave as a conformal fluid in 2 spatial dimensions just like that of the M2-brane. The fact that the thermodynamic properties of the D1-brane theory is similar to the M2-brane was noted earlier in [26] and [27]. In [26], it was noted that the entropy density of the D1-brane behaves like that of the M2-brane ${ }^{5}$, while in [27] the speed of sound in the D1-brane matter was evaluated holographically just using thermodynamics of the D1-brane and shown to be the same as given in (5.2). It will be interesting to understand this coincidence since the D1-brane gravity solution is certainly not of the M2-brane form.

From comparing the dissipative part of the dispersion relation in (4.46) and (5.1) we see that

$$
\frac{\xi}{\epsilon+P}=\frac{\alpha}{3}=\frac{L^{3}}{6 r_{0}^{2}}=\frac{1}{4 \pi T} .
$$

In the absence of chemical potentials, we have the following thermodynamic relation

$$
\epsilon+P=T s
$$

Substituting the above equation in (5.3), we find that

$$
\frac{\xi}{s}=\frac{1}{4 \pi} .
$$

Using (2.10) and the definition of $L$ from (2.2), the entropy density can be written in terms of the field theory variables and temperature as

$$
s=\frac{2^{4} \pi^{\frac{5}{2}}}{3^{3}} \frac{N^{2} T^{2}}{\sqrt{\lambda}} .
$$

Then the bulk viscosity is given by

$$
\xi=\frac{2^{6} \pi^{\frac{7}{2}}}{3^{3}} \frac{N^{2} T^{2}}{\sqrt{\lambda}} .
$$

Let us now compare the result in (5.5) with the ratio of bulk viscosity to entropy density for Dp-branes with $p \geq 2$. Using the results of [15] for Dp-branes with $p \geq 2$ we have

$$
\frac{\xi}{s}=\frac{\xi}{\eta} \frac{\eta}{s}=\frac{1}{4 \pi} \frac{2(3-p)^{2}}{p(9-p)} .
$$

Note that the above equation is valid $p \geq 2$, since the method used by [15] is valid only for $p \geq 2$. But using the result (5.5), it is clear that the formula for $\xi / s$ obtained for $p \geq 2$ continues to hold for also $p=1$.

\footnotetext{
${ }^{5}$ See below equation 2.17 in 26
} 


\section{$5.2 \xi / s$ using the Kubo's formula}

As a cross check of our calculations, we use the Kubo's formula to obtain the ratio of bulk viscosity to entropy density for the D1-branes. In $1+1$ dimensions, Kubo's formula for bulk viscosity is given by (see for instance in [20])

$$
\begin{aligned}
\xi & =\lim _{\omega \rightarrow 0} \frac{1}{\omega} \int_{0}^{\infty} d t \int d z e^{i \omega t}\left\langle\left[T_{z z}(x), T_{z z}(0)\right]\right\rangle . \\
& =\lim _{\omega \rightarrow 0} \frac{i}{\omega} G_{z z, z z}(\omega, q=0) .
\end{aligned}
$$

As we have seen in section 4.4, the two point function of the stress tensor from gravity is given by

$$
G_{z z, z z}=\frac{\omega^{4}}{\left(\omega^{2}-q^{2}\right)^{2}} G_{B}(\omega, q), \quad G_{B}=-\frac{1}{16 \pi G_{3}} \frac{r_{0}^{6}}{L^{7}} \frac{D}{C} .
$$

Here, the coefficients $\mathrm{C}$ and $\mathrm{D}$ are related to the asymptotic expansion of the solution near the boundary at $u \rightarrow 0$ given by

$$
Z_{P}=C(1+\ldots)+D u^{3}(1+\ldots) .
$$

For the sound mode in (4.44), we find that

$$
\begin{aligned}
& C=\frac{\left(-4 i \alpha \omega^{3}+4 i \alpha \omega q^{2}+6 \omega^{2}-3 q^{2}\right)}{9(2 i \alpha \omega-3)} \\
& D=\frac{\omega\left[9 i \omega q^{2}+8 i \alpha^{2} \omega\left(\omega^{2}-q^{2}\right)^{2}-12 \alpha\left(2 q^{4}-3 q^{2} \omega^{2}+\omega^{4}\right)\right]}{54(3 i+2 \alpha \omega)\left(q^{2}-\omega^{2}\right)}
\end{aligned}
$$

In limit $q \rightarrow 0$, we obtain

$$
C \rightarrow \frac{-2 \omega^{2}}{9} \quad D \rightarrow \frac{-2 i \alpha \omega^{3}}{27} \quad \frac{D}{C} \rightarrow \frac{i \alpha \omega}{3}
$$

and hence

$$
G_{z z, z z}(\omega, 0)=G_{B}(\omega, 0)=-\frac{1}{16 \pi G_{3}} \frac{6 r_{0}^{6}}{L^{7}} \frac{i \alpha \omega}{3}
$$

Now using the temperature and the entropy density of the D1-branes solution given in (2.10), we can write the two point function as

$$
G_{z z, z z}(\omega, 0)=-T s \frac{\alpha \omega}{3}=-i \frac{\omega s}{4 \pi} .
$$

Substituting this result for the two point function into Kubo's formula in (5.9), we obtain the ratio

$$
\frac{\xi}{s}=\frac{1}{4 \pi}
$$

This agrees with that obtained from examining the dissipative part of the sound mode in (5.5) and therefore is a consistency check on our calculations. 


\subsection{Universality of the ratio $\xi / s$}

\section{(i) $\xi / s$ for the fundamental string}

We have seen that in the range of temperatures $\sqrt{\lambda} N^{-\frac{2}{3}}<<T<<\sqrt{\lambda}$ for which the D1-brane solution can be trusted, the ratio $\xi / s$ is given by the ideal value $1 / 4 \pi$. We now show that the ratio continues to be $1 / 4 \pi$ for the temperature range $\sqrt{\lambda} N^{-1}<<T<<\sqrt{\lambda} N^{-\frac{2}{3}}$ for which the fundamental string solution can be trusted. From (2.13), we see that the only difference between the F1-string solution and that of the D1-brane solution is that of the dilaton profile. In fact the F1-string solution can be obtained from the D1-brane solution by replacing $\phi \rightarrow-\phi$. On compactifying to 3 dimensions just as for the D1-brane case, one obtains a consistent truncation of the equations of motion. The equations of motion can be derived from the action in 3 dimensions given in (4.3) but with

$$
\beta=\frac{16}{9}, \quad \text { and } \mathcal{P}=-\frac{24}{L^{2}} e^{-\frac{4}{3} \phi} .
$$

The F1-string solution in 10 dimension given in (2.13) reduces to

$$
\begin{aligned}
d s^{2} & =-c_{T}(r)^{2} d t^{2}+c_{X}(r)^{2} d z^{2}+c_{R}(r)^{2} d r^{2}, \\
\phi & =3 \log \left(\frac{r}{L}\right) .
\end{aligned}
$$

with $c_{T}, c_{X}, c_{R}$ and $f$ given by (4.7) just as for the D1-brane case. Note that the only change from the D1-brane case is that of the sign of the dilaton.

The analysis of the linearized perturbation and obtaining the equation for the sound mode in section 4.1 and 4.2 just depended on the fact that the background is of the form in (5.18) and radially symmetric. Thus the equation for the sound mode for the F1-string solution continues to be (5.20) which is given by

$$
Z_{P}^{\prime \prime}+\left[\ln ^{\prime}\left(\frac{c_{T} c_{X}}{c_{R}}\right)-2 \frac{A_{\varphi}^{\prime}}{A_{\varphi}}\right] Z_{P}^{\prime}-\left[\frac{c_{R}^{2}}{c_{T}^{2}}\left(A_{t}-\omega^{2}\right)+2 \frac{A_{\varphi}^{\prime}}{A_{\varphi}} \ln ^{\prime}\left(\frac{c_{X}}{c_{T}}\right)\right] Z_{P}=0 .
$$

In the above equation, the only dependence on the dilaton profile is due to the ratio $\frac{A_{\varphi}^{\prime}}{A_{\varphi}}$. From the definition of $A_{\varphi}$ in (4.25), we see that this ratio is given by

$$
\frac{A_{\varphi}^{\prime}}{A_{\varphi}}=\frac{\left[A_{H} \ln ^{\prime}\left(c_{X}\right)\right]^{\prime}}{A_{H} \ln ^{\prime}\left(c_{X}\right)}-\frac{\phi^{\prime \prime}}{\phi^{\prime}} .
$$

Since $A_{H}$ just depends on the components of the metric (4.23), the only difference for the F1-string can arise from the ratio $\phi^{\prime \prime} / \phi^{\prime}$. But it is easy to see from (5.18),

that this ratio is same as that of the D1-brane case. Therefore the ratio $\frac{A_{\varphi}^{\prime}}{A_{\varphi}}$ for the F1-string remains identical to that of the D1-brane case. Thus, the equation of the 
sound mode $Z_{P}$ is the same for the F1-string. Now the rest of the analysis to extract the dispersion relation for the sound mode proceeds identical to that of the D1-brane case. This results in the ratio

$$
\frac{\xi}{s}=\frac{1}{4 \pi}
$$

for the F1-string background. We have thus extended the evaluation of the bulk viscosity to the temperature range $\sqrt{\lambda} N^{-1}<<T<<\sqrt{\lambda}$ for the $U(N)$ gauge theory with 16 supercharges in $1+1$ dimensions.

\section{(ii) $\xi / s$ for D1-branes at cones over Sasaki-Einstein 7-manifolds}

Here we show that the ratio of the bulk viscosity to entropy density for theories of D1-branes at a cone over Sasaki-Einstein 7 -manifolds is also given by $1 / 4 \pi$ in the regimes given by (1.2). The near horizon geometry of the corresponding supergravity solution is given by

$$
\begin{aligned}
d s^{2} & =H^{-\frac{3}{4}}(r)\left(-f(r) d t^{2}+d x_{1}^{2}\right)+H^{\frac{1}{4}}(r)\left(\frac{d r^{2}}{f(r)}+r^{2} d S_{X_{7}}^{2}\right), \\
e^{\phi(r)} & =H(r)^{\frac{1}{2}} \\
F_{7} & =6 L^{6} \omega_{X_{7}} .
\end{aligned}
$$

where

$$
H(r)=\left(\frac{L}{r}\right)^{6}, \quad f(r)=1-\left(\frac{r_{0}}{r}\right)^{6} .
$$

and $d S_{X_{7}}^{2}$ stands for the metric of the 7 dimensional Sasaki-Einstein manifold $X_{7}$, and $\omega_{X_{7}}$ is its volume form. Note that the only way this background differs from D1-brane at flat space is by the replacement of the 7-sphere by the 7-dimensional Sasaki-Einstein manifold. To be explicit here, we write down the metrics of two 7-dimension Sasaki-Einstein manifolds known as $Q^{1,1,1}$ and $M^{1,1,1}$ in the literature. The metric of $Q^{1,1,1}$ is given by 28

$$
d S_{Q^{1,1,1}}^{2}=\frac{1}{16}\left(d \psi-\sum_{i=1}^{3} \cos \theta_{i} d \phi_{i}\right)^{2}+\frac{1}{8} \sum_{i=1}^{3}\left(d \theta_{i}^{2}+\sin ^{2} \theta_{i} d \phi_{i}^{2}\right),
$$

while the metric of $M^{1,1,1}$ is given by 29

$$
\begin{aligned}
d S_{M^{1,1,1}}^{2}= & \frac{1}{64}\left(d \tau+3 \sin ^{2} \mu \sigma_{3}+2 \cos \theta_{2} d \phi_{2}\right)^{2} \\
& +\frac{3}{4}\left(d \mu^{2}+\frac{1}{4} \sin ^{2} \mu\left(\sigma_{1}^{2}+\sigma_{2}^{2}+\cos ^{2} \mu \sigma_{3}^{2}\right)\right) \\
& +\frac{1}{8}\left(d \theta_{2}^{2}+\sin ^{2} \theta_{2} d \phi_{2}^{2}\right),
\end{aligned}
$$

where

$$
\sigma_{1}=d \theta_{1}, \quad \sigma_{2}=\sin \theta_{1} d \phi_{1}, \quad \sigma_{3}=\left(d \psi+\cos \theta_{1} d \phi_{1}\right)
$$


Similar to the case of flat space we now dimensionally reduce to 3 dimensions using the ansatz

$$
\begin{aligned}
d s_{10}^{2} & =e^{-14 B(r)} g_{\mu \nu}(x) d x^{\mu} d x^{\nu}+e^{2 B(r)} L^{2} d S_{X_{7}}^{2} \\
& =e^{-14 B(r)}\left(-c_{T}^{2}(r) d t^{2}+c_{X}^{2}(r) d x_{1}^{2}+c_{R}^{2} d r^{2}\right)+e^{2 B(r)} L^{2} d S_{X_{7}}^{2}
\end{aligned}
$$

Again one can verify just as in the case of flat space the identification

$$
B(r)=-\frac{1}{12} \phi(r)
$$

provides a consistent reduction provided the 7-form flux through the Sasaki-Einstein manifold is held constant. The equations of motion reduce to the Einstein-dilaton system in 3 dimensions. The coefficient of the potential of the dilaton in this system is dependent on the the Ricci-scalar of the Sasaki-Einstein manifold. For the metric $L^{2} d S_{X_{7}}^{2}$ when $X_{7}$ is either $Q^{1,1,1}$ or $M^{1,1,1}$ the Ricci-scalar is given by

$$
R\left(X_{7}\right)=\frac{42}{L^{2}}
$$

This is same as that of the 7-sphere, which implies the coefficient of the dilaton potential will remain the same in the effective action. Note that the Ricci-scalar for the general $Y^{(p, q)}$ Sasaki-Einstein 7-manifolds constructed in 30] and recently studied in [31] is also given by $(5.29)^{6}$. Taking all this into consideration, the effective action in 3-dimensions is given by

$$
I=\frac{1}{16 \pi \tilde{G}_{3}} \int d^{3} x \sqrt{-g}\left(R(g)-\frac{8}{9} \partial_{\mu} \phi \partial^{\mu} \phi+\frac{24}{L^{2}} e^{\frac{4}{3} \phi}\right),
$$

where

$$
\frac{1}{\tilde{G}_{3}}=\frac{L^{7} \operatorname{Vol}\left(X_{7}\right)}{G_{10}} .
$$

We see apart from the definition of the 3 dimensional Newton's constant, the effective action remains the same as that of D1-branes in flat space. The background values of $c_{T}(r), c_{X}(r), c_{R}(r)$ and $\phi(r)$ is also same as that of D1-branes in flat space. Therefore the quasi-normal mode analysis remains identical and the ratio of the bulk viscosity to the entropy density remains the same. The analysis for the case of the F1-strings at cones over Sasaki-Einstein 7-manifolds is also identical to that of F1-strings in flat space and yields the same ratio of bulk viscosity by entropy density.

The gauge theory of D1-branes at cones over Sasaki-Einstein 7-manifolds is different from that of the $S U(N)$ theory with 16 supercharges for the case of D1-branes in flat space. The number of supersymmetries and the matter content of the theory is different. Inspite of this for the temperature range (1.2), the theories of D1-branes at cones over Sasaki-Einstein 7 -manifolds have the same value of $\xi / s=1 / 4 \pi$ as that of D1-branes in flat space.

\footnotetext{
${ }^{6}$ See below equation 2.6 of [31].
} 


\section{Conclusions}

We have shown that the ratio of bulk viscosity to the entropy density for the $S U(N)$ gauge theory with 16 supercharges in $1+1$ dimensions on the D1-branes in the temperature range $\sqrt{\lambda} N^{-1}<<T<<\sqrt{\lambda}$ is given by $1 / 4 \pi$. For temperatures outside this range, the D1-brane gauge theory flows to a conformal field theory. We therefore expect the bulk viscosity to entropy density of this theory to vanish for $T<<\sqrt{\lambda} N^{-1}$ and $T>>\sqrt{\lambda}$. A technical result of our analysis is the equation for the sound mode given in (4.36) for any Einstein-dilaton system in 3 dimensions given by the Lagrangian (4.3) which admits a radially symmetric solution of the form (4.5) and a dilaton profile determined by the dilaton equation of motion.

We have also seen that for the theory of D1-branes at cones over Sasaki-Einstein 7-manifolds the near horizon geometry dimensionally reduced to 3-dimensions is identical to that of D1-branes in flat space. This implies that the ratio of bulk viscosity to entropy density is given by $1 / 4 \pi$. At this point, it is perhaps worthwhile to investigate other $1+1$ dimensional systems which admit gravity duals with different near horizon geometry. There are two possible interesting geometries one could explore: One can turn on a R-charge along the Cartan directions of the $S O(8) \mathrm{R}$ symmetry of the D1-brane theory. This results in a R-charged black hole in the Einstein-dilaton-Maxwell system in 3-dimensions. The near horizon geometry of such black holes in 3-dimensions is different and it will be interesting to evaluate the ratio of bulk viscosity to entropy density in this geometry. Another interesting geometry in 3-dimensions is that of the D1-D5 system. This geometry is conformal, however one could turn on a relevant operator in the orbifold theory of the D1-D5 system which will render it non-conformal. The holographic dual geometry to such a system will necessarily have a non-trivial dilaton profile and therefore a non-trivial bulk viscosity. This system will fall into the general Einstein-dilaton system studied in this paper, with an specific dilaton potential. We expect the dual geometry to be radially symmetric since one can possibly choose the relevant deformation to be translationally invariant along the brane directions. From the analysis in this paper we can then conclude that the equation for the sound mode is given by (4.36). It is tempting to speculate that the ratio of bulk viscosity to entropy density for this system will also by $1 / 4 \pi$. It will be interesting to construct this dual geometry for the deformed D1-D5 system explicitly and verify this conjecture.

One of our motivations to study holographic duals to hydrodynamics in $1+1$ dimensions is the possibility of taking the non-relativisitc limit to obtain the Burger's equation ${ }^{7}$ and study turbulence in one spatial dimension. However the scaling put forward in 32, 33 to obtain the non-relativisitc limit results in velocity of sound

\footnotetext{
${ }^{7}$ Burger's equation is the non-relativistic Navier-Stokes equation in one spatial dimension given by $\partial_{t} v+v \partial_{x} v=\xi \partial_{x}^{2} v$, where $v$ is the velocity.
} 
being infinite. This leads to hydrodynamics with divergenceless velocity flow,

$$
\partial_{i} v^{i}=0 .
$$

In one spatial dimension, this implies a trivial solution for the velocity field. The velocity field is constant in both space and time. It will be interesting to explore the possibility of taking other non-relativistic limits which can lead to the Burger's equation.

Note added: After completion of this work, we noticed the preprint [34] which has some overlap with this paper.

\section{Acknowledgments}

We would like to especially thank Shiraz Minwalla for useful suggestions and insights at various stages in this project. We also thank Rajesh Gopakumar and Nemani Suryanarayana for discussions. J.R.D thanks the organizers of the Monsoon Workshop on String Theory (2008) and ICTS, TIFR for the stimulating environment which resulted in this project. M.M thanks the Centre for High Energy Physics, IISc. for hospitality during which part of the work was done. Finallly we would like to thank the people of India for supporting research in fundamental physics.

\section{A. Consistency of the constraint equations}

Note that the equations (4.18)-(4.20) form 3 constraints for the 4 dynamical equations (4.14)-(4.17). These constraints must therefore be consistent with the dynamical equations. That is, on evolving the constraints by the dynamical equations, one should not generate new constraints. In this appendix, we show that by differentiating the 3 constraints in (4.18) to (4.20) and reducing them again to first order equations using the dynamical equations in (4.14) to (4.17), one does not generate any new constraints. This allows us to conclude that the constraints are consistent with the dynamical equations and they reduce the number of dynamical variables to just one, which we have identified as the sound mode $Z_{P}$.

We start with writing down 2 equations which we will use in our analysis. We define

$$
Z_{0}=A_{t} H_{t t}+2 q \omega H_{t z}+\omega^{2} H_{z z},
$$

where $A_{t}=q^{2} \frac{c_{T}^{2}}{c_{X}^{2}}$. Its first derivative is

$$
Z_{0}^{\prime}=A_{t}^{\prime} H_{t t}+A_{t} H_{t t}^{\prime}+2 \omega q H_{t z}^{\prime}+\omega^{2} H_{z z}^{\prime}
$$


Using the constraint equations, one can remove $H_{t t}^{\prime}$ and $H_{z z}^{\prime}$ in favour of $H_{t z}^{\prime}$. Then the expression reduces to

$Z_{0}^{\prime}=\left\{A_{t}^{\prime}+A_{t} \ln ^{\prime}\left(\frac{c_{X}}{c_{T}}\right)\right\} H_{t t}-\omega^{2} \ln ^{\prime}\left(\frac{c_{X}}{c_{T}}\right) H_{z z}-2 \omega q \ln ^{\prime}\left(\frac{c_{X}}{c_{T}}\right) H_{t z}+\left(A_{t}-\omega^{2}\right) \beta \phi^{\prime} \varphi$.

Next we write down an equation obtained by differentiating $Z_{P}$.

$$
\begin{aligned}
Z_{P}^{\prime} & =Z_{0}^{\prime}+A_{\varphi} \varphi^{\prime}+A_{\varphi}^{\prime} \varphi \\
& =-\ln ^{\prime}\left(\frac{c_{X}}{c_{T}}\right) Z_{P}+\left\{\ln ^{\prime}\left(\frac{c_{X}}{c_{T}}\right) A_{\varphi}+\left(A_{t}-\omega^{2}\right) \beta \phi^{\prime}+A_{\varphi}^{\prime}\right\} \varphi+A_{\varphi} \varphi^{\prime} .
\end{aligned}
$$

Here we have used the equation (A.3). Equation (A.4) can also be obtained by adding $A_{t}$ times (4.18) and $\omega^{2}$ times (4.19).

To check the consistency of the constraint equations, we first differentiate them, then substitute the dynamical equations and reduce the equation to an equation with at the most first derivatives. We then show that we do not obtain any new constraints. We start with the equation (4.19). We get

$$
\begin{aligned}
& -\ln ^{\prime}\left(\frac{c_{T} c_{X}^{2}}{c_{R}}\right) H_{t t}^{\prime}+H_{z z}^{\prime} \ln ^{\prime}\left(c_{T}\right)+\frac{\omega c_{X}^{2}}{q c_{T^{2}}} \ln ^{\prime}\left(\frac{c_{R}}{c_{X} c_{T}}\right) H_{t z}^{\prime}-\ln ^{\prime \prime}\left(\frac{c_{X}}{c_{T}}\right) H_{t t}+\frac{c_{R}^{2}}{c_{T}^{2}} Z_{P} \\
& +\left(2 c_{R}^{2} \frac{\partial \mathcal{P}}{\partial \phi}-\frac{c_{R}^{2}}{c_{T}^{2}} A_{\varphi}-\beta \phi^{\prime \prime}\right) \varphi-\beta \phi^{\prime} \varphi^{\prime}=0 .
\end{aligned}
$$

When we use (4.30) on the combination of equations $(4.5)-(4.20)+\ln ^{\prime}\left(\frac{c_{T} c_{X}}{c_{R}}\right)$ times (4.19), we get

$$
\Omega \varphi=0
$$

where

$$
\Omega=c_{R}^{2} \mathcal{P}^{\prime}-\ln ^{\prime}\left(\frac{c_{T} c_{X}}{c_{R}}\right) \beta \phi^{\prime}-\beta \phi^{\prime \prime} .
$$

Now (A.6) is consistent for any fluctuation $\varphi$ because $\Omega=0$, since the back ground statisfies the dilaton equation of motion (4.11). Thus the equation (A.5) is consistent with equations of motion.

Next we differentiate (4.18) and use the dynamical equations to remove the second derivatives. Doing this, we get the equation

$$
\begin{aligned}
& -\ln ^{\prime}\left(\frac{c_{T} c_{X}^{2}}{c_{R}}\right) H_{z z}^{\prime}-\frac{q}{\omega} \ln ^{\prime}\left(\frac{c_{X}^{3}}{c_{T} c_{R}}\right) H_{t z}^{\prime}+\left\{\ln ^{\prime}\left(c_{X}\right)-\frac{A_{t}}{\omega^{2}} \ln ^{\prime}\left(\frac{c_{X}}{c_{T}}\right)\right\} H_{t t}^{\prime} \\
& -\frac{1}{\omega^{2}}\left(A_{t} \ln ^{\prime} \frac{c_{X}}{c_{T}}\right)^{\prime} H_{t t}+\left\{\frac{1}{\omega^{2}} \ln ^{\prime \prime}\left(\frac{c_{X}}{c_{T}}\right)-\frac{c_{R}^{2}}{c_{T}^{2}}\right\} Z_{P}+\frac{1}{\omega^{2}} \ln ^{\prime}\left(\frac{c_{X}}{c_{T}}\right) Z_{P}^{\prime} \\
& +\left[-\frac{1}{\omega^{2}}\left\{A_{\varphi} \ln ^{\prime}\left(\frac{c_{X}}{c_{T}}\right)\right\}^{\prime}+\beta \phi^{\prime \prime}+\frac{c_{R}^{2}}{c_{T}^{2}} A_{\varphi}-2 c_{R}^{2} \frac{\partial \mathcal{P}}{\partial \phi}\right] \varphi \\
& +\left\{-\frac{1}{\omega^{2}} A_{\varphi} \ln \left(\frac{c_{X}}{c_{T}}\right)+\beta \phi^{\prime}\right\} \varphi^{\prime}=0 .
\end{aligned}
$$


Note that using equation (4.30), one can show that

$$
\ln ^{\prime \prime}\left(\frac{c_{X}}{c_{T}}\right)-\left[\ln ^{\prime}\left(\frac{c_{X}}{c_{T}}\right)\right]^{2}=\ln ^{\prime}\left(\frac{c_{T}}{c_{X}}\right) \ln ^{\prime}\left(\frac{c_{X}^{2}}{c_{R}}\right)
$$

Using (A.9) on the combination of equations $($ A.8) -4.20$)+\frac{A_{t}}{\omega^{2}} \ln ^{\prime}\left(\frac{c_{X}}{c_{T}}\right)$ times $(4.19)$ $+\ln ^{\prime}\left(\frac{c_{X}^{2}}{c_{R}}\right)$ times (4.18), one obtains

$$
-\Omega \varphi=0
$$

Hence, the equation (A.8) can be written as a linear combination of earlier constraint equations.

The derivative of third constraint equation (4.20) gives us

$$
\begin{aligned}
& H_{z z}^{\prime}\left[\ln ^{\prime \prime}\left(c_{T}\right)-\ln ^{\prime} c_{T} \ln ^{\prime}\left(\frac{c_{T} c_{X}^{2}}{c_{R}}\right)-\ln ^{\prime} c_{X} \ln ^{\prime} c_{T}+\frac{\beta}{2} \phi^{\prime 2}\right] \\
& +H_{t t}^{\prime}\left[-\ln ^{\prime \prime}\left(c_{X}\right)+\ln ^{\prime} c_{X} \ln ^{\prime}\left(\frac{c_{T}^{2} c_{X}}{c_{R}}\right)+\ln ^{\prime} c_{X} \ln ^{\prime} c_{T}-\frac{\beta}{2} \phi^{\prime 2}\right] \\
& +\varphi^{\prime}\left[\beta \phi^{\prime} \ln ^{\prime}\left(\frac{c_{T} c_{X}}{c_{R}}\right)-\beta \phi^{\prime \prime}-\frac{c_{R}^{2}}{c_{T}^{2}} A_{\varphi}+c_{R}^{2} \mathcal{P}^{\prime}\right] \\
& +c_{R}^{2} \varphi\left[-2 \mathcal{P}^{\prime} \ln ^{\prime}\left(\frac{c_{T} c_{X}}{c_{R}}\right)+\beta \phi^{\prime}\left(\frac{\omega^{2}}{c_{T}^{2}}-\frac{q^{2}}{c_{X}^{2}}\right)+\frac{A_{\varphi}}{c_{T}^{2}} \ln ^{\prime}\left(\frac{c_{T}^{3} c_{X}}{c_{R}^{2}}\right)-\frac{A_{\varphi}^{\prime}}{c_{T}^{2}}\right] \\
& +\frac{c_{R}^{2}}{c_{T}^{2}}\left\{Z_{P}^{\prime}-\ln ^{\prime}\left(\frac{c_{T}^{3} c_{X}}{c_{R}^{2}}\right) Z_{P}\right\}=0 .
\end{aligned}
$$

We use equation $\Omega=0$ to remove the $\beta \phi^{\prime \prime} \varphi^{\prime}$ term and equation (A.4) to remove $Z_{P}^{\prime}$. Next we add $2 \ln ^{\prime}\left(\frac{c_{T} c_{X}}{c_{R}}\right)$ times $(4.20)$ to it. We note that equation (4.30) can also be written as

$$
\ln ^{\prime \prime} c_{T}+\ln ^{\prime} c_{T} \ln ^{\prime}\left(\frac{c_{T} c_{X}}{c_{R}}\right)=\ln ^{\prime \prime} c_{X}+\ln ^{\prime} c_{X} \ln ^{\prime}\left(\frac{c_{T} c_{X}}{c_{R}}\right)
$$

Using this equation with the above manipulations, we obtain

$$
\left[\ln ^{\prime \prime} c_{T}+\ln ^{\prime} c_{T} \ln ^{\prime}\left(\frac{c_{T} c_{X}}{c_{R}}\right)-2 \ln ^{\prime} c_{T} \ln ^{\prime} c_{X}+\frac{\beta}{2} \phi^{\prime 2}\right]\left(H_{z z}-H_{t t}\right)^{\prime}=0
$$

The term in the square bracket is a combination of Einstein equations of motion, namely equation (4.9)- $\frac{1}{2}(4.10$, hence it vanishes. This implies that A.11) can be written as a linear combination of the three basic constraints upto equations of motion. 


\section{B. Identities from background field equations}

(i) Derivation of equation (4.39)

First we will derive equation (4.32) from the background equations of motion. The $\left(\omega^{2}\right)$ and $\left(q^{2}\right)$ part of the equation should independently vanish. Since

$$
A_{\varphi}^{\prime}=-\frac{\phi^{\prime \prime}}{\phi^{\prime}} A_{\varphi}+\frac{2}{\phi^{\prime}}\left[A_{t}\left\{\ln ^{\prime \prime} c_{T}+2 \ln ^{\prime} c_{T} \ln ^{\prime}\left(\frac{c_{T}}{c_{X}}\right)\right\}-\omega^{2} \ln ^{\prime \prime} c_{X}\right]
$$

we need to show

$$
\begin{gathered}
\frac{2}{\phi^{\prime}}\left[\frac{\beta \phi^{\prime 2}}{2}+2 \ln ^{\prime} c_{T} \ln ^{\prime}\left(\frac{c_{T}}{c_{X}}\right)+\ln ^{\prime \prime} c_{T}+\ln ^{\prime} c_{T} \ln ^{\prime}\left(\frac{c_{X}}{c_{T} c_{R}}\right)\right]=0 \\
\text { and } \quad \frac{2}{\phi^{\prime}}\left[\frac{\beta \phi^{\prime 2}}{2}+\ln ^{\prime \prime} c_{X}+\ln ^{\prime} c_{X} \ln ^{\prime}\left(\frac{c_{X}}{c_{T} c_{R}}\right)\right]=0 .
\end{gathered}
$$

Using equations (4.9) and (4.8), we obtain

$$
\begin{aligned}
& \ln ^{\prime \prime} c_{T}=\ln ^{\prime} c_{T} \ln ^{\prime}\left(\frac{c_{R}}{c_{T}}\right)-\frac{\beta}{4} \phi^{2}-\frac{c_{R}^{2}}{2} \mathcal{P}, \\
& \ln ^{\prime \prime} c_{X}=\ln ^{\prime} c_{X} \ln ^{\prime}\left(\frac{c_{R}}{c_{X}}\right)-\frac{\beta}{4} \phi^{\prime 2}-\frac{c_{R}^{2}}{2} \mathcal{P} .
\end{aligned}
$$

Replacing $\ln ^{\prime \prime} c_{T}\left(\ln ^{\prime \prime} c_{X}\right)$ in equation $[\mathrm{B} .2]([\mathrm{B} .3])$, we find that the left hand side vanishes due to equation (4.10).

(ii) Derivation of (4.33)

Next, we will derive relation (4.33). The $\left(\omega^{2}\right)$ and $\left(q^{2}\right)$ part of the equation should be independently satisfied. They are

$$
\begin{array}{r}
G^{\prime}+2 c_{R}^{2} \mathcal{P}^{\prime}+2 \frac{c_{R}^{2} \mathcal{P}^{\prime \prime}}{\beta \phi^{\prime}} \ln ^{\prime} c_{X}+G\left[\frac{2 \phi^{\prime \prime}}{\phi^{\prime}}+\ln ^{\prime}\left(\frac{c_{T} c_{X}}{c_{R}^{3}}\right)\right]=0, \\
4 \beta \phi^{\prime} \ln ^{\prime}\left(\frac{c_{T}}{c_{X}}\right)+K^{\prime}+2 c_{R}^{2} \mathcal{P}^{\prime}+\frac{2 c_{R}^{2} \mathcal{P}^{\prime \prime}}{\beta \phi^{\prime}} \ln ^{\prime} c_{T}+K \ln ^{\prime}\left(\frac{c_{T}^{3}}{c_{X} c_{R}^{3}}\right)+\frac{2 \phi^{\prime \prime}}{\phi^{\prime}} K=0 .
\end{array}
$$

where

$$
\begin{aligned}
G & =\frac{2}{\phi^{\prime}}\left[\ln ^{\prime \prime} c_{X}-\frac{\phi^{\prime \prime}}{\phi^{\prime}} \ln ^{\prime} c_{X}\right], \\
K & =\frac{2}{\phi^{\prime}}\left[\ln ^{\prime \prime} c_{T}+2 \ln ^{\prime} c_{T} \ln ^{\prime}\left(\frac{c_{T}}{c_{X}}\right)-\frac{\phi^{\prime \prime}}{\phi^{\prime}} \ln ^{\prime} c_{T}\right] .
\end{aligned}
$$

To show (B.6), we first evaluate $G^{\prime}$.

$$
G^{\prime}=-\frac{2 \phi^{\prime \prime}}{\phi^{\prime}} G+\frac{2}{\phi^{\prime}}\left[\ln ^{(3)} c_{X}-\frac{\phi^{(3)}}{\phi^{\prime}} \ln ^{\prime} c_{X}\right]
$$


Using equations (4.8) and (4.11), we can write

$$
\begin{aligned}
\ln ^{(3)} c_{X}-\frac{\phi^{(3)}}{\phi^{\prime}} \ln ^{\prime} c_{X}= & \ln ^{\prime \prime} c_{X} \ln ^{\prime}\left(\frac{c_{R}}{c_{X}}\right)+\ln ^{\prime} c_{X} \ln ^{\prime \prime} c_{T}+\frac{\phi^{\prime \prime}}{\phi^{\prime}} \ln ^{\prime} c_{X} \ln ^{\prime}\left(\frac{c_{T} c_{X}}{c_{R}}\right) \\
& -\frac{2 c_{R}^{2} \mathcal{P}^{\prime}}{\beta \phi^{\prime}} \frac{c_{X}^{\prime}}{c_{X}} \frac{c_{R}^{\prime}}{c_{R}}-\frac{c_{R}^{2} \mathcal{P}^{\prime \prime}}{\beta} \ln ^{\prime} c_{X}-c_{R}^{2} \mathcal{P} \ln ^{\prime} c_{R} \\
& +\frac{\phi^{\prime}}{2}\left\{-2 c_{R}^{2} \mathcal{P}^{\prime}+\beta \phi^{\prime} \ln ^{\prime}\left(\frac{c_{T} c_{X}}{c_{R}}\right)\right\}
\end{aligned}
$$

Then the left hand side of equation (B.6) becomes

$$
\begin{aligned}
L H S= & G \ln ^{\prime}\left(\frac{c_{T} c_{X}}{c_{R}^{3}}\right)+\frac{2}{\phi^{\prime}}\left[\frac{\beta \phi^{\prime 2}}{2} \ln ^{\prime}\left(\frac{c_{T} c_{X}}{c_{R}}\right)+\ln ^{\prime \prime} c_{X} \ln ^{\prime}\left(\frac{c_{R}}{c_{X}}\right)+\ln ^{\prime} c_{X} \ln ^{\prime \prime} c_{T}\right. \\
& \left.+\frac{\phi^{\prime \prime}}{\phi^{\prime}} \ln ^{\prime} c_{X} \ln ^{\prime}\left(\frac{c_{T} c_{X}}{c_{R}}\right)-\frac{2 c_{R}^{2} \mathcal{P}^{\prime}}{\beta \phi^{\prime}} \frac{c_{R}^{\prime}}{c_{R}} \frac{c_{X}^{\prime}}{c_{X}}-c_{R}^{2} \mathcal{P} \ln ^{\prime} c_{R}\right] \\
= & \frac{2}{\phi^{\prime}}\left[\frac{\beta \phi^{\prime 2}}{2} \ln ^{\prime}\left(\frac{c_{T} c_{X}}{c_{R}}\right)+\ln ^{\prime \prime} c_{X} \ln ^{\prime}\left(\frac{c_{T}}{c_{R}^{2}}\right)+\ln ^{\prime} c_{X} \ln ^{\prime \prime} c_{T}\right. \\
& \left.+\frac{2 \phi^{\prime \prime}}{\phi^{\prime}} \ln ^{\prime} c_{X} \ln ^{\prime} c_{R}-\frac{2 c_{R}^{2} \mathcal{P}^{\prime}}{\beta \phi^{\prime}} \frac{c_{R}^{\prime}}{c_{R}} \frac{c_{X}^{\prime}}{c_{X}}-c_{R}^{2} \mathcal{P} \ln ^{\prime} c_{R}\right]
\end{aligned}
$$

Using equations (4.10) and (4.11), one can show that

$$
\begin{aligned}
-\left(\ln ^{\prime \prime} c_{T} \ln ^{\prime} c_{X}+\ln ^{\prime} c_{T} \ln ^{\prime \prime} c_{X}\right) & =\frac{1}{2}\left[c_{R}^{2} \mathcal{P}-\frac{\beta}{2} \phi^{\prime 2}\right]^{\prime} \\
& =c_{R}^{2} P \ln ^{\prime} c_{R}+\frac{\beta \phi^{\prime 2}}{2} \ln ^{\prime}\left(\frac{c_{T} c_{X}}{c_{R}}\right) .
\end{aligned}
$$

Then we obtain

$$
L H S=\frac{2 \ln ^{\prime} c_{R}}{\phi^{\prime}}\left[-2 \ln ^{\prime \prime} c_{X}+\frac{2 \phi^{\prime \prime}}{\phi^{\prime}} \ln ^{\prime} c_{X}-\frac{2 c_{R}^{2} \mathcal{P}^{\prime}}{\beta \phi^{\prime}} \frac{c_{X}^{\prime}}{c_{X}}-2 c_{R}^{2} \mathcal{P}\right] .
$$

Using equation (B.5) to replace $\ln ^{\prime \prime} c_{X}$ and equation (4.11) to replace $\phi^{\prime \prime}$, the LHS then vanishes due to equation (4.10).

To show (B.7), we first evaluate

$$
\begin{aligned}
K^{\prime}= & -2 \frac{\phi^{\prime \prime}}{\phi^{\prime}} K+\frac{2}{\phi^{\prime}}\left[\ln ^{\prime \prime} c_{T}\left\{\ln ^{\prime}\left(\frac{c_{R}}{c_{T}^{2}}\right)+2 \ln ^{\prime}\left(\frac{c_{T}}{c_{X}}\right)\right\}+\frac{c_{T}^{\prime}}{c_{T}} \frac{c_{R}^{\prime}}{c_{R}}+\frac{\beta \phi^{\prime 2}}{2} \ln ^{\prime}\left(\frac{c_{T} c_{X}}{c_{R}}\right)\right. \\
& -c_{R}^{2} \mathcal{P}^{\prime} \phi^{\prime}-c_{R}^{2} \mathcal{P} \ln ^{\prime} c_{R}+2 \ln ^{\prime} c_{T} \ln ^{\prime \prime}\left(\frac{c_{T}}{c_{X}}\right)+\frac{2 \phi^{\prime \prime}}{\phi^{\prime}} \ln ^{\prime} c_{T} \ln ^{\prime}\left(\frac{c_{T}}{c_{X}}\right) \\
& \left.+\ln ^{\prime} c_{T}\left\{\frac{\phi^{\prime \prime}}{\phi^{\prime}} \ln ^{\prime}\left(\frac{c_{T} c_{X}}{c_{R}}\right)+\ln ^{\prime \prime}\left(\frac{c_{T} c_{X}}{c_{R}}\right)-\frac{2 c_{R}^{2} \mathcal{P}^{\prime}}{\beta \phi^{\prime}} \ln ^{\prime} c_{R}-\frac{c_{R}^{2} \mathcal{P}^{\prime \prime}}{\beta}\right\}\right] .
\end{aligned}
$$

Using equations (4.11) and (4.9), we can write

$$
\begin{aligned}
\frac{c_{R}^{2} \mathcal{P}^{\prime}}{\beta} \ln ^{\prime} c_{T} \ln ^{\prime} c_{R}= & 2 \phi^{\prime \prime} \frac{c_{T}^{\prime}}{c_{T}} \frac{c_{R}^{\prime}}{c_{R}} \\
& +2 \ln ^{\prime}\left(\frac{c_{T} c_{X}}{c_{R}}\right)\left\{\ln ^{\prime \prime} c_{T}+\left(\frac{c_{T}^{\prime}}{c_{T}}\right)^{2}+\frac{\beta}{2} \phi^{\prime 2}+\frac{c_{R}^{2} \mathcal{P}}{2}\right\} .
\end{aligned}
$$


Using it, we get

$$
\begin{aligned}
K^{\prime}= & -\frac{2 \phi^{\prime \prime}}{\phi^{\prime}} K-2 c_{R}^{2} \mathcal{P}^{\prime}-\frac{2 c_{R}^{2} \mathcal{P}^{\prime \prime}}{\beta \phi^{\prime}} \ln ^{\prime} c_{T} \\
& +\frac{2}{\phi^{\prime}}\left[\ln ^{\prime} c_{T}\left\{\frac{\phi^{\prime \prime}}{\phi^{\prime}} \ln ^{\prime}\left(\frac{c_{T}^{3}}{c_{X} c_{R}^{3}}\right)-\ln ^{\prime \prime} c_{X}-2 \ln ^{\prime} c_{T} \ln ^{\prime}\left(\frac{c_{T} c_{X}}{c_{R}}\right)\right\}\right. \\
& \left.+\ln ^{\prime \prime} c_{T} \ln ^{\prime}\left(\frac{c_{T} c_{R}^{3}}{c_{X}^{4}}\right)-c_{R}^{2} \mathcal{P} \ln ^{\prime}\left(c_{T} c_{X}\right)\right]
\end{aligned}
$$

The left hand side of the equation (B.7) then becomes

$$
\begin{aligned}
\text { LHS }= & \frac{2}{\phi^{\prime}}\left[\ln ^{\prime \prime} c_{T} \ln ^{\prime}\left(\frac{c_{T}^{4}}{c_{X}^{5}}\right)+2 \ln ^{\prime}\left(\frac{c_{T}}{c_{X}}\right) \ln ^{\prime} c_{T} \ln ^{\prime}\left(\frac{c_{T}^{3}}{c_{X} c_{R}^{3}}\right)+2 \beta \phi^{2} \ln ^{\prime}\left(\frac{c_{T}}{c_{X}}\right)\right. \\
& \left.-c_{R}^{2} \mathcal{P} \ln ^{\prime}\left(c_{T} c_{X}\right)-\ln ^{\prime} c_{T} \ln ^{\prime \prime} c_{X}-2\left(\ln ^{\prime} c_{T}\right)^{2} \ln ^{\prime}\left(\frac{c_{T} c_{X}}{c_{R}}\right)\right] .
\end{aligned}
$$

Using equation (B.13), we get

$$
\begin{aligned}
L H S= & \frac{2}{\phi^{\prime}}\left[\ln ^{\prime}\left(\frac{c_{T}}{c_{X}}\right)\left\{4 \ln ^{\prime \prime} c_{T}+2 \ln ^{\prime} c_{T} \ln ^{\prime}\left(\frac{c_{T}^{3}}{c_{X} c_{R}^{3}}\right)+2 \beta \phi^{\prime 2}\right\}\right. \\
& \left.+\ln ^{\prime}\left(\frac{c_{T} c_{X}}{c_{R}}\right)\left\{-c_{R}^{2} \mathcal{P}+\frac{\beta \phi^{\prime 2}}{2}-2\left(\ln ^{\prime} c_{T}\right)^{2}\right\}\right] .
\end{aligned}
$$

Using equation (B.4), we get

$$
\begin{aligned}
L H S= & \frac{2}{\phi^{\prime}}\left[\ln ^{\prime}\left(\frac{c_{T}}{c_{X}}\right)\left\{\beta \phi^{\prime 2}-2 c_{R}^{2} \mathcal{P}+2 \ln ^{\prime} c_{T} \ln ^{\prime}\left(\frac{c_{T}}{c_{X} c_{R}}\right)\right\}\right. \\
& \left.+\ln ^{\prime}\left(\frac{c_{T} c_{X}}{c_{R}}\right)\left\{-c_{R}^{2} \mathcal{P}+\frac{\beta \phi^{\prime 2}}{2}-2\left(\ln ^{\prime} c_{T}\right)^{2}\right\}\right] \\
= & \frac{2}{\phi^{\prime}}\left[\ln ^{\prime}\left(\frac{c_{T}}{c_{X}}\right)\left\{4 \ln ^{\prime} c_{T} \ln ^{\prime} c_{X}+2 \ln ^{\prime} c_{T} \ln ^{\prime}\left(\frac{c_{T}}{c_{X} c_{R}}\right)\right\}\right. \\
& \left.+\ln ^{\prime}\left(\frac{c_{T} c_{X}}{c_{R}}\right)\left\{2 \ln ^{\prime} c_{T} \ln ^{\prime} c_{X}-2\left(\ln ^{\prime} c_{T}\right)^{2}\right\}\right] \\
= & 0=R H S,
\end{aligned}
$$

where we used equation (4.10) to get middle step.

\section{References}

[1] G. Policastro, D. T. Son, and A. O. Starinets, The shear viscosity of strongly coupled $N=4$ supersymmetric Yang-Mills plasma, Phys. Rev. Lett. 87 (2001) 081601, hep-th/0104066. 
[2] G. Policastro, D. T. Son, and A. O. Starinets, From AdS/CFT correspondence to hydrodynamics, JHEP 09 (2002) 043, hep-th/0205052.

[3] G. Policastro, D. T. Son, and A. O. Starinets, From AdS/CFT correspondence to hydrodynamics. II: Sound waves, JHEP 12 (2002) 054, hep-th/0210220.

[4] C. P. Herzog, The hydrodynamics of M-theory, JHEP 12 (2002) 026, hep-th/0210126.

[5] P. Kovtun, D. T. Son, and A. O. Starinets, Holography and hydrodynamics: Diffusion on stretched horizons, JHEP 10 (2003) 064, hep-th/0309213.

[6] C. P. Herzog, The sound of M-theory, Phys. Rev. D68 (2003) 024013, hep-th/0302086.

[7] P. Kovtun, D. T. Son, and A. O. Starinets, Viscosity in strongly interacting quantum field theories from black hole physics, Phys. Rev. Lett. 94 (2005) 111601, hep-th/0405231.

[8] D. T. Son and A. O. Starinets, Viscosity, Black Holes, and Quantum Field Theory, Ann. Rev. Nucl. Part. Sci. 57 (2007) 95-118, arXiv:0704.0240.

[9] S. Bhattacharyya, V. E. Hubeny, S. Minwalla, and M. Rangamani, Nonlinear Fluid Dynamics from Gravity, JHEP 02 (2008) 045, arXiv:0712.2456.

[10] R. Baier, P. Romatschke, D. T. Son, A. O. Starinets, and M. A. Stephanov, Relativistic viscous hydrodynamics, conformal invariance, and holography, JHEP 04 (2008) 100, arXiv:0712.2451.

[11] A. Buchel and J. T. Liu, Universality of the shear viscosity in supergravity, Phys. Rev. Lett. 93 (2004) 090602, hep-th/0311175.

[12] H. J. Boonstra, K. Skenderis, and P. K. Townsend, The domain wall/QFT correspondence, JHEP 01 (1999) 003, hep-th/9807137.

[13] N. Itzhaki, J. M. Maldacena, J. Sonnenschein, and S. Yankielowicz, Supergravity and the large $N$ limit of theories with sixteen supercharges, Phys. Rev. D58 (1998) 046004, [hep-th/9802042].

[14] I. Kanitscheider, K. Skenderis, and M. Taylor, Precision holography for non-conformal branes, JHEP 09 (2008) 094, arXiv:0807.3324.

[15] J. Mas and J. Tarrio, Hydrodynamics from the Dp-brane, JHEP 05 (2007) 036, hep-th/0703093.

[16] P. Benincasa, A. Buchel, and A. O. Starinets, Sound waves in strongly coupled non-conformal gauge theory plasma, Nucl. Phys. B733 (2006) 160-187, hep-th/0507026. 
[17] P. Benincasa and A. Buchel, Hydrodynamics of Sakai-Sugimoto model in the quenched approximation, Phys. Lett. B640 (2006) 108-115, hep-th/0605076.

[18] A. Buchel, Bulk viscosity of gauge theory plasma at strong coupling, Phys. Lett. B663 (2008) 286-289, arXiv:0708.3459.

[19] L. D. Landau and E. M. Lifshitz, Course of Theoretical Physics. Vol. 6: Fluid Mechanics, . Elsevier. (1984) 539p.

[20] L. P. Kadanoff and P. C. Martin, Hydrodynamic equations and correlation functions, Ann. Phys. 24 (1963) 419.

[21] M. Cvetic, H. Lu, and C. N. Pope, Consistent Kaluza-Klein sphere reductions, Phys. Rev. D62 (2000) 064028, hep-th/0003286.

[22] P. K. Kovtun and A. O. Starinets, Quasinormal modes and holography, Phys. Rev. D72 (2005) 086009, hep-th/0506184.

[23] R.-G. Cai and N. Ohta, Surface counterterms and boundary stress-energy tensors for asymptotically non-anti-de Sitter spaces, Phys. Rev. D62 (2000) 024006, hep-th/9912013.

[24] A. Batrachenko, J. T. Liu, R. McNees, W. A. Sabra, and W. Y. Wen, Black hole mass and Hamilton-Jacobi counterterms, JHEP 05 (2005) 034, hep-th/0408205.

[25] T. Wiseman and B. Withers, Holographic renormalization for coincident Dp-branes, JHEP 10 (2008) 037, arXiv:0807.0755].

[26] A. W. Peet and J. Polchinski, UV/IR relations in AdS dynamics, Phys. Rev. D59 (1999) 065011, hep-th/9809022].

[27] D. Mateos, R. C. Myers, and R. M. Thomson, Thermodynamics of the brane, JHEP 05 (2007) 067, hep-th/0701132.

[28] D. N. Page and C. N. Pope, Which compactifications of D=11 supergravity are stable?, Phys. Lett. B144 (1984) 346.

[29] D. N. Page and C. N. Pope, Stability analysis of compactifications of D $=11$ supergravity With $S U(3) \times S U(2) \times U(1)$ symmetry, Phys. Lett. B145 (1984) 337 .

[30] J. P. Gauntlett, D. Martelli, J. F. Sparks, and D. Waldram, A new infinite class of Sasaki-Einstein manifolds, Adv. Theor. Math. Phys. 8 (2006) 987-1000, hep-th/0403038.

[31] D. Martelli and J. Sparks, Notes on toric Sasaki-Einstein seven-manifolds and $A d S_{4} / C F T_{3}, J H E P 11$ (2008) 016, arXiv:0808.0904.

[32] I. Fouxon and Y. Oz, Conformal Field Theory as Microscopic Dynamics of Incompressible Euler and Navier-Stokes Equations, arXiv:0809.4512. 
[33] S. Bhattacharyya, S. Minwalla, and S. R. Wadia, The Incompressible Non-Relativistic Navier-Stokes Equation from Gravity, arXiv:0810.1545.

[34] I. Kanitscheider and K. Skenderis, Universal hydrodynamics of non-conformal branes, arXiv:0901.1487. 